TRANSACTIONS OF THE

AMERICAN MATHEMATICAL SOCIETY

Volume 358, Number 3, Pages 1285-1315

S 0002-9947(05)03742-6

Article electronically published on May 26, 2005

\title{
COMPLEX SYMMETRIC OPERATORS AND APPLICATIONS
}

\author{
STEPHAN RAMON GARCIA AND MIHAI PUTINAR
}

\begin{abstract}
We study a few classes of Hilbert space operators whose matrix representations are complex symmetric with respect to a preferred orthonormal basis. The existence of this additional symmetry has notable implications and, in particular, it explains from a unifying point of view some classical results. We explore applications of this symmetry to Jordan canonical models, selfadjoint extensions of symmetric operators, rank-one unitary perturbations of the compressed shift, Darlington synthesis and matrix-valued inner functions, and free bounded analytic interpolation in the disk.
\end{abstract}

\section{INTRODUCTION}

The simultaneous diagonalization and spectral analysis of two Hermitian forms goes back to the origins of Hilbert space theory and, in particular, to the spectral theorem for self-adjoint operators. Even today the language of forms is often used when dealing with unbounded operators (see [31, 43]). The similar theory for a Hermitian and a nondefinite sesquilinear form was motivated by the Hamiltonian mechanics of strings or continuous media models; from a mathematical point of view this theory leads to Hilbert spaces with a complex linear $J$-involution and the associated theory of $J$-unitary and $J$-contractive operators (see for instance [19, 31, 41). Less studied, but not less important, is the simultaneous analysis of a pair consisting of a Hermitian form and a bilinear form; this framework has appeared quite early in function theory $([9,48,51])$, functional analysis ([37]), and elasticity theory (17). Some of the main results in this direction were estimates derived from variational principles for eigenvalues of symmetric matrices (such as Grunsky's or Friedrichs' inequalities).

The present note was motivated by the observation that all scalar (Jordan) models in operator theory are complex symmetric with respect to a well-chosen orthonormal basis; cf. [8, 39]. Put into the context of a pair of a Hermitian and a bilinear form, this remark reveals an extra symmetry of these model operators, shared rather surprisingly by quite a few other basic classes of operators such as normal, Hankel, compressed Toeplitz, and some Volterra operators. It is no accident that exactly this symmetry appears in one of Siegel's matrix realizations of Cartan domains 49 .

Received by the editors February 21, 2004 and, in revised form, May 10, 2004.

2000 Mathematics Subject Classification. Primary 30D55, 47A15.

Key words and phrases. Complex symmetric operators, interpolation, self-adjoint extension, Takagi factorization, shift operators, inner functions, Darlington synthesis, Clark perturbations, Jordan operators, Volterra operators.

The second author was supported in part by NSF Grant DMS \#0100367. 
Technically speaking, we consider a complex Hilbert space $\mathcal{H}$ and an antilinear, isometric involution $C$ on it. A bounded linear operator $T$ is called $C$-symmetric if $C T=T^{*} C$. This is equivalent to the symmetry of $T$ with respect to the bilinear form $[f, g]=\langle f, C g\rangle$. It is easy to show that there exists an orthonormal basis $\left(e_{i}\right)_{i \in I}$ of $\mathcal{H}$ which is left invariant by $C: C e_{i}=e_{i}$. With respect to the basis $\left(e_{i}\right)_{i \in I}$, $C$-symmetry is simply complex symmetry of the associated matrix. Already at this general level the symmetry $C T=T^{*} C$ has strong effects on the spectral picture of $T$; for instance, the generalized eigenspaces $\operatorname{Ker}(T-\lambda)^{p}$ and $\operatorname{Ker}\left(T^{*}-\bar{\lambda}\right)^{p}$ are antilinearly isometrically isomorphic via $C$. Thus a Fredholm $C$-symmetric operator has zero index.

The examples of $C$-symmetric operators are numerous and quite diverse. Besides the expected normal operators, certain Volterra and Toeplitz operators are $C$-symmetric. For example, consider a finite Toeplitz matrix with complex entries:

$$
\left[\begin{array}{cccc}
a_{0} & a_{1} & \ldots & a_{n} \\
a_{-1} & a_{0} & \ldots & a_{n-1} \\
\vdots & \vdots & & \vdots \\
a_{-n} & a_{-n+1} & \ldots & a_{0}
\end{array}\right]
$$

The symmetry with respect to the second diagonal leaves this matrix invariant, and this is exactly the $C$-symmetry noticed and exploited a long time ago by Schur and Takagi [51]. At the level of functional models, the symmetry

$$
f \mapsto C f:=\overline{f z} \varphi
$$

maps the standard model space $\mathcal{H}_{\varphi}=H^{2} \ominus \varphi H^{2}$ onto itself and makes the compression of the unilateral shift a $C$-symmetric operator. Above $H^{2}$ is the Hardy space of the disk and $\varphi$ is a nonconstant inner function.

The applications of $C$-symmetry we discuss in this article can be grouped into the following categories: extension and dilation results, rank one perturbations of Jordan operators, matrix-valued inner functions and free interpolation theory in the disk.

The first three of these subjects are interconnected by a simple matrix completion observation. Namely, every $C$-symmetric operator admits $C$-symmetric extensions and dilations. At the level of real symmetric operators and their self-adjoint extensions this remark goes back to von Neumann [37, and also explicitly appears in the computations of M.G. Krein 28. The same phenomenon is present in Clark's unitary perturbations of Jordan operators, or in the study of real Volterra operators pursued by the Ukrainian school, 27] and also [7, 24, 25, 36. It was this last group of researchers who investigated for the first time $C$-symmetries of various linear systems appearing in mathematical physics or engineering. At the abstract level, we observe that every $C$-symmetric contraction has a $C$-symmetric Sz.-Nagy unitary dilation.

We also examine the canonical model spaces $\mathcal{H}_{\varphi}$ and the compressed Toeplitz operators carried by them from the viewpoint of $C$-symmetry. In particular, we show how to use Clark's theory [11] to produce complex symmetric matrix realizations for Jordan operators. Also $C$-symmetry turns out to be fundamental in understanding the structure of inner $2 \times 2$ matrix-valued functions in the disk. This 
subject is related to Darlington's synthesis problem in systems theory, and our approach offers a concrete parametrization of all solutions to the scalar Darlington problem.

A different theme, touched upon in Section 7, is the classical free interpolation problem in the unit disk. For an interpolating Blaschke product $\varphi$ and the associated involution $C$ on the model space $\mathcal{H}_{\varphi}$, we show the identity between a Fourier type orthogonal decomposition with respect to the bilinear form $[\cdot, \cdot]:=\langle\cdot, C \cdot\rangle$ :

$$
f=\sum_{n=1}^{\infty} \frac{\left[f, e_{n}\right]}{\left[e_{n}, e_{n}\right]} e_{n}
$$

for $f$ in $\mathcal{H}_{\varphi}$ and the standard division and interpolation results. The novelty in the above representation formula is the orthogonality of its terms with respect to the new bilinear form.

The last section contains a couple of simple examples of quotients of Hilbert spaces of analytic functions defined on domains of $\mathbb{C}^{n}$. They illustrate the possible complications arising from the generalization of the complex symmetry of Jordan operators to several complex variables.

The present paper consists of a blend of a few distinct bodies: elementary observations and examples, new proofs to old results, new interpretations of classical results, hints to other predictable applications and some original facts. We have the feeling that complex symmetric matrices have not exhausted their surprising ramifications.

The bibliography at the end of the article contains, besides the needed references for the technical proofs, a few sources of old and new ideas exploiting the extra complex symmetry of various classes of linear operators. We have not aimed at completeness and we apologize for omissions or inaccuracies.

\section{2. $C$-SYMMETRIES}

2.1. Preliminaries. Let $\mathcal{H}$ denote a separable Hilbert space and $C$ an isometric antilinear involution of $\mathcal{H}$. By isometric we mean that $\langle f, g\rangle=\langle C g, C f\rangle$ for all $f, g$ belonging to $\mathcal{H}$.

A typical example of a symmetry $C$ as above is the complex conjugation of functions belonging to a Sobolev space of a domain in $\mathbb{R}^{n}$. Another example is the term by term complex conjugation

$$
C\left(z_{0}, z_{1}, z_{2}, \ldots\right)=\left(\overline{z_{0}}, \overline{z_{1}}, \overline{z_{2}}, \ldots\right)
$$

of a vector in $l^{2}(\mathbb{N})$. As proved below, this example is typical.

Lemma 1. If $C$ is an isometric antilinear involution on the Hilbert space $\mathcal{H}$, then there exists an orthonormal basis $e_{n}$ such that $e_{n}=C e_{n}$ for all $n$. Each $h$ in $\mathcal{H}$ can be written uniquely in the form $h_{1}+i h_{2}$ where $h_{1}=C h_{1}$ and $h_{2}=C h_{2}$. Moreover, $\|h\|^{2}=\left\|h_{1}\right\|^{2}+\left\|h_{2}\right\|^{2}$.

Proof. Let $e_{n}$ be an orthonormal basis for the real Hilbert subspace $(I+C) \mathcal{H}$ of $\mathcal{H}$. Hence every vector in $(I+C) \mathcal{H}$ is of the form $\sum_{n=0}^{\infty} a_{n} e_{n}$ where $a_{n}$ is a square-summable sequence of real numbers. Noting the decomposition

$$
\begin{aligned}
h & =\frac{1}{2}(I+C) h+i \frac{1}{2 i}(I-C) h \\
& =\frac{1}{2}(I+C) h+i \frac{1}{2}(I+C)(-i h)
\end{aligned}
$$


we see that every $h$ in $\mathcal{H}$ lies in the complex linear span of $(I+C) \mathcal{H}$ and hence $e_{n}$ is an orthonormal basis for $\mathcal{H}$. The remainder of the proposition follows immediately from (1) and a straightforward computation using the isometric property of $C$.

As a consequence of the preceding proposition, we will sometimes refer to $C$ as a conjugation operator. Although the existence of a self-conjugate basis or $C$-real basis is guaranteed by Lemma 1, it is sometimes difficult to explicitly describe one. Nevertheless, there are certain circumstances (see for instance Section 6) when such an explicit computation is possible and indeed fruitful.

Example 1. Consider the typical nontrivial invariant subspace for the backward shift operator on the classical Hardy space $H^{2}$. It is well known (see [10] for example) that the proper, nontrivial invariant subspaces for the backward shift operator are precisely the subspaces

$$
\mathcal{H}_{\varphi}:=H^{2} \ominus \varphi H^{2}
$$

where $\varphi$ is a nonconstant inner function. Since

$$
\langle\overline{f z} \varphi, \overline{z h}\rangle=\langle\varphi h, f\rangle=0
$$

and

$$
\langle\overline{f z} \varphi, \varphi h\rangle=\langle\overline{z h}, f\rangle=0
$$

for each $f$ in $\mathcal{H}_{\varphi}$ and $h$ in $H^{2}$, we see that

$$
C f:=\overline{f z} \varphi
$$

defines a conjugation operator on $\mathcal{H}_{\varphi}$. In particular, we see that $\overline{f z} \varphi$, despite its appearance, is the boundary function for an $H^{2}$ function.

Even at this basic level, $C$-symmetry is a powerful concept. The decomposition (11) yields an explicit function-theoretic characterization of $\mathcal{H}_{\varphi}$ [21 and hence of functions which are pseudocontinuable of bounded type (see [14, 46]). By Lemma 1, it suffices to classify self-conjugate functions. Suppose that $\zeta$ is a point on $\partial \mathbb{D}$ such that $\varphi$ has a nontangential limiting value at $\zeta$ of unit modulus and $c$ is a unimodular constant satisfying $c^{2}=\bar{\zeta} \varphi(\zeta)$. By (3), a self-conjugate function $f$ satisfies $f=\bar{f} z \varphi$ a.e on $\partial \mathbb{D}$ and hence $f(z)=\operatorname{cr}(z) K_{\zeta}(z)$ where

$$
K_{\zeta}(z)=\frac{1-\overline{\varphi(\zeta)} \varphi(z)}{1-\bar{\zeta} z}
$$

and $r(z)$ is a function in the Smirnov class $N^{+}$whose boundary values are real a.e. on $\partial \mathbb{D}$. Such functions are described explicitly in $[20,23,34$.

We remark that some of this can be generalized to the de Branges-Rovnyak setting, although we do not pursue that course in detail here. If $b$ is an extreme point of the unit ball of $H^{\infty}$ (that is, if $\log \left(1-\left|b\left(e^{i t}\right)\right|\right)$ is not integrable [15, Thm. 7.9]) and $\mu_{b}$ is the measure on $\partial \mathbb{D}$ whose Poisson integral is the real part of $(1+b) /(1-b)$, then one can define a conjugation operator on the associated de Branges-Rovnyak space $H(b)$ that naturally corresponds to complex conjugation in $L^{2}\left(\mu_{b}\right)$ [35, Sect. 9].

Example 2. Consider a bounded, positive continuous weight $\rho$ on the interval $[-1,1]$, symmetric with respect to the midpoint of the interval: $\rho(t)=\rho(-t)$ for 
$t$ in $[0,1]$. Let $P_{n}$ be the associated orthogonal polynomials, normalized by the conditions

$$
\int_{-1}^{1} P_{n}(t)^{2} \rho(t) d t=1, \quad \lim _{x \rightarrow \infty} P_{n}(x) / x^{n}=1 .
$$

Due to their uniqueness, these polynomials have real coefficients and satisfy

$$
P_{n}(-t)=(-1)^{n} P_{n}(t)
$$

for all $t$. Thus,

$$
e_{n}(t)=i^{n} P_{n}(t)
$$

for $n \geq 0$ is a $C$-real basis for $L^{2}([-1,1], \rho d t)$ with respect to the symmetry $C f(t):=$ $\overline{f(-t)}$.

2.2. Reproducing kernels. Let us assume now that $\mathcal{H}$ is a reproducing kernel Hilbert space (of scalar-valued functions) on a space $X$. If $\mathcal{H}$ is endowed with an isometric conjugation operator $C$, then

$$
C f(w)=\left\langle C f, K_{w}\right\rangle=\left\langle C K_{w}, f\right\rangle
$$

for $f$ in $\mathcal{H}$. Therefore the conjugate kernel $Q_{w}:=C K_{w}$ reproduces the values of $C f(w)$ via the formula $C f(w)=\left\langle Q_{w}, f\right\rangle$. This is to be expected, since $f \mapsto C f(w)$ is a bounded antilinear functional on $\mathcal{H}$.

While the reproducing kernel is antisymmetric $\left(K_{w}(z)=\overline{K_{z}(w)}\right.$ for all $z, w$ in $X)$, the conjugate kernel $Q_{w}$ is symmetric in $z$ and $w$ :

$$
Q_{w}(z)=\left\langle C K_{w}, K_{z}\right\rangle=\left\langle C K_{z}, K_{w}\right\rangle=Q_{z}(w) .
$$

Indeed, if $e_{n}$ is a $C$-real basis, then

$$
Q_{w}(z)=\sum_{n=1}^{\infty} e_{n}(z) e_{n}(w)
$$

for all $z, w$ in $X$. This follows from the well-known formula

$$
K_{w}(z)=\sum_{n=1}^{\infty} e_{n}(z) \overline{e_{n}(w)}
$$

which holds for any orthonormal basis $e_{n}$.

Example 3. Let us return to the subspace $\mathcal{H}_{\varphi}$ and the conjugation operator $C$ of Example 1. The reproducing kernel of $\mathcal{H}_{\varphi}$ is

$$
K_{w}(z)=\frac{1-\overline{\varphi(w)} \varphi(z)}{1-\bar{w} z}
$$

where $z, w$ belong to the unit disk $\mathbb{D}$. The corresponding conjugate kernel is

$$
Q_{w}(z)=\frac{\varphi(z)-\varphi(w)}{z-w} .
$$

We will refer to these two functions often in the following pages. 
2.3. The bilinear form $[\cdot, \cdot]$. Each conjugation operator $C$ is equivalent, via the Riesz representation theorem, to a symmetric bilinear form

$$
[f, g]:=\langle f, C g\rangle
$$

defined for $f, g$ in $\mathcal{H}$. This form is nondegenerate and isometric, in the sense that

$$
\sup _{\|g\|=1}|[f, g]|=\|f\|
$$

for all $f$ in $\mathcal{H}$. Conversely, if a nondegenerate, bilinear, symmetric, and isometric form $[\cdot, \cdot \cdot]$ is given, then there exists an isometric antilinear operator $C$ on $\mathcal{H}$ satisfying (6). Since $\|C f\|=\|f\|$, we infer that

$$
\langle f, f\rangle=\langle C f, C f\rangle=[C f, f]=[f, C f]=\left\langle f, C^{2} f\right\rangle,
$$

hence $C^{2}=I$ and $C$ is a conjugation operator.

\section{3. $C$-SYMMETRIC OPERATORS}

3.1. Basic properties. The main object of study in this article is a linear (usually bounded) operator $T$ acting on a separable, complex Hilbert space $\mathcal{H}$ and satisfying

$$
C T=T^{*} C,
$$

where $C$ is a conjugation operator on $\mathcal{H}$. We say then that $T$ is $C$-symmetric and refer to $(\mathcal{H}, T, C)$ as a $C$-symmetric triple.

For a fixed $C$, we consider the set

$$
C^{\circ}:=\left\{T \in B(\mathcal{H}): C T=T^{*} C\right\}
$$

of all $C$-symmetric operators. Clearly, $C^{\circ}$ is a $*$-closed linear manifold in $B(\mathcal{H})$ containing the identity. It is a small exercise to check that $C^{\circ}$ is closed in the norm, weak operator, and strong operator topologies and that the adjoint is continuous on $C^{\circ}$ with respect to all three topologies.

The next proposition contains a few remarks based on the definition of $C$ symmetry. The proofs are simple and left to the reader.

Proposition 1. Let $(\mathcal{H}, T, C)$ be a C-symmetric triple. Then:

(1) $T$ is left invertible if and only if $T$ is right invertible. If $T^{-1}$ exists, then $T^{-1}$ is also $C$-symmetric.

(2) $\operatorname{Ker} T$ is trivial if and only if $\operatorname{Ran} T$ is dense in $\mathcal{H}$.

(3) If $T$ is Fredholm, then ind $T=0$.

(4) $p(T)$ is $C$-symmetric for any polynomial $p(z)$.

(5) For each $\lambda$ and $n \geq 0$, the map $C$ establishes an antilinear isometric isomorphism between $\operatorname{Ker}(T-\lambda I)^{n}$ and $\operatorname{Ker}\left(T^{*}-\bar{\lambda} I\right)^{n}$.

The preceding proposition has several immediate spectral consequences. The last statement implies that the point spectra of $T$ and $T^{*}$ correspond under complex conjugation. Since $C$ is isometric, the same correspondence holds for the approximate point spectra of $T$ and $T^{*}$, as well as other spectral structures.

We first examine a few examples of $C$-symmetric matrices. We will later examine more sophisticated examples of $C$-symmetric operators and then the present finiterank examples will be instructive. 
Example 4. One of the simplest, and perhaps most important, families of $C$ symmetric operators are the finite Jordan blocks. Let $\lambda$ be a complex number and consider the Jordan block $J_{n}(\lambda)$ of order $n$ corresponding to $\lambda$. In other words,

$$
J_{n}(\lambda):=\left(\begin{array}{cccccc}
\lambda & 1 & & & & \\
& \lambda & 1 & & & \\
& & \lambda & & & \\
& & & \ddots & & \\
& & & & \lambda & 1 \\
& & & & & \lambda
\end{array}\right) .
$$

If $C_{n}$ denotes the isometric antilinear operator

$$
C_{n}\left(z_{1}, z_{2}, \ldots, z_{n}\right):=\left(\overline{z_{n}}, \ldots, \overline{z_{2}}, \overline{z_{1}}\right)
$$

on $\mathbb{C}^{n}$, then one readily computes that $\left(\mathbb{C}^{n}, J_{n}(\lambda), C_{n}\right)$ is a $C_{n}$-symmetric triple for any $\lambda$. In particular, the operators $J_{n}(\lambda)$ for $\lambda \in \mathbb{C}$ are simultaneously $C_{n^{-}}$ symmetric. Since a direct sum of finite rank Jordan blocks is clearly $C$-symmetric, any operator on a finite dimensional space is similar to a $C$-symmetric operator (see also [18, 30]).

The proper notion of equivalence for $C$-symmetric operators, or more appropriately $C$-symmetric triples, is unitary equivalence. Given a $C$-symmetric triple $\left(\mathcal{H}_{1}, T_{1}, C_{1}\right)$ and a unitary operator $U: \mathcal{H}_{1} \rightarrow \mathcal{H}_{2}$, we obtain a new $C$-symmetric triple $\left(\mathcal{H}_{2}, T_{2}, C_{2}\right)$ where $T_{2}=U T_{1} U^{*}$ and $C_{2}=U C_{1} U^{*}$. Indeed, since $C_{1} T_{1}=$ $T_{1}^{*} C_{1}$, we see that

$$
C_{2} T_{2}=\left(U C_{1} U^{*}\right)\left(U T_{1} U^{*}\right)=\left(U T_{1}^{*} U^{*}\right)\left(U C_{1} U^{*}\right)=T_{2}^{*} C_{2} .
$$

We say that two triples $\left(\mathcal{H}_{1}, T_{1}, C_{1}\right)$ and $\left(\mathcal{H}_{2}, T_{2}, C_{2}\right)$ are equivalent if there exists a unitary operator $U: \mathcal{H}_{1} \rightarrow \mathcal{H}_{2}$ such that $T_{2}=U T_{1} U^{*}$ and $C_{2}=U T_{1} U^{*}$. This is clearly an equivalence relation.

Example 5. For any complex number $a$ the matrix

$$
T=\left(\begin{array}{ll}
1 & a \\
0 & 0
\end{array}\right)
$$

defines a $C$-symmetric operator on $\mathbb{C}^{2}$. By performing a unitary change of coordinates, we may assume that $a$ is real. Since $C$ must map the one-dimensional eigenspaces of $T$ corresponding to the eigenvalues 0 and 1 onto the corresponding eigenspaces of $T^{*}$, one can readily verify that $\left(\mathbb{C}^{2}, T, C\right)$ is a $C$-symmetric triple where

$$
C\left(\begin{array}{l}
z_{1} \\
z_{2}
\end{array}\right)=\left(\begin{array}{cc}
\frac{1}{\sqrt{1+a^{2}}} & \frac{a}{\sqrt{1+a^{2}}} \\
\frac{a}{\sqrt{1+a^{2}}} & -\frac{1}{\sqrt{1+a^{2}}}
\end{array}\right)\left(\begin{array}{l}
\overline{z_{1}} \\
\frac{z_{2}}{2}
\end{array}\right) .
$$

Example 6. All $2 \times 2$ complex matrices define $C$-symmetric operators on $\mathbb{C}^{2}$, with a proper choice of the $C$-symmetry. By unitary equivalence, it suffices to consider upper triangular $2 \times 2$ matrices. Since $T-\lambda I$ is $C$-symmetric if and only if $T$ is, we need only appeal to Example 5 to draw the desired conclusion. 
Example 7. The preceding example indicates that we must look to $3 \times 3$ matrices to find the simplest operators that are not $C$-symmetric. The matrix

$$
T=\left(\begin{array}{lll}
1 & a & 0 \\
0 & 0 & b \\
0 & 0 & 1
\end{array}\right)
$$

is $C$-symmetric if and only if $|a|=|b|$. If $|a|=|b|$, then $T$ is unitarily equivalent to

$$
\left(\begin{array}{lll}
1 & a & 0 \\
0 & 0 & a \\
0 & 0 & 1
\end{array}\right)
$$

which is $C$-symmetric with respect to $C\left(z_{1}, z_{2}, z_{3}\right)=\left(\overline{z_{3}}, \overline{z_{2}}, \overline{z_{1}}\right)$.

Now suppose that $|a| \neq|b|$ and observe that $T$ has eigenvalues $0,1,1$ but does not have two linearly independent eigenvectors corresponding to the eigenvalue 1 . To see that $T$ is not $C$-symmetric, note that

$$
\left|\left\langle e_{0}, e_{1}\right\rangle\right| \neq\left|\left\langle f_{1}, f_{0}\right\rangle\right|
$$

whenever $e_{0}, e_{1}$ and $f_{0}, f_{1}$ are unit eigenvectors (corresponding to the eigenvalues 0 and 1 , respectively) for $T$ and $T^{*}$, respectively. Take, for instance,

$$
e_{0}=\left(\begin{array}{c}
-\frac{a}{s} \\
\frac{1}{s} \\
0
\end{array}\right), \quad e_{1}=\left(\begin{array}{l}
1 \\
0 \\
0
\end{array}\right), \quad f_{0}=\left(\begin{array}{c}
0 \\
\frac{1}{t} \\
-\frac{b}{t}
\end{array}\right), \quad f_{1}=\left(\begin{array}{l}
0 \\
0 \\
1
\end{array}\right) \text {, }
$$

where $s=\sqrt{1+|a|^{2}}$ and $t=\sqrt{1+|b|^{2}}$.

The preceding example shows that not all finite rank operators are $C$-symmetric. A geometric explanation lies in the fact that the angles between the eigenspaces of a $C$-symmetric operator $T$ must coincide (via $C$ ) with the complex conjugates of the corresponding angles between the eigenspaces of $T^{*}$. This does not occur for general finite rank $T$.

3.2. Complex symmetric matrices. We can characterize $C$-symmetric operators in terms of certain matrix representations. Let $(\mathcal{H}, T, C)$ be a $C$-symmetric triple and let $e_{n}$ be the orthonormal basis for $\mathcal{H}$ provided by Lemma 1 . With respect to the basis $e_{n}$, the matrix associated to $T$ is complex symmetric: $\left\langle T e_{n}, e_{m}\right\rangle=$ $\left\langle T e_{m}, e_{n}\right\rangle$ for all $n, m$. Indeed, this follows from a straightforward computation based on the equation $C T=T^{*} C$ and the isometric property of $C$ :

$$
\left\langle T e_{n}, e_{m}\right\rangle=\left\langle C e_{m}, C T e_{n}\right\rangle=\left\langle e_{m}, T^{*} C e_{n}\right\rangle=\left\langle T e_{m}, C e_{n}\right\rangle=\left\langle T e_{m}, e_{n}\right\rangle .
$$

Thus we have proved the following proposition.

Proposition 2. Let $T$ be a bounded linear operator on a Hilbert space $\mathcal{H}$. The following conditions are equivalent:

(1) $T$ is $C$-symmetric for an isometric antilinear involution $C$.

(2) There exists an isometric, symmetric bilinear form $[f, g]$ on $\mathcal{H}$ with respect to which $T$ is symmetric.

(3) There exists an orthonormal basis of $\mathcal{H}$ with respect to which $T$ has a symmetric matrix representation. 
Before proceeding to our next example, we briefly remark that the set $C^{\circ}$ defined by (77) is not closed under multiplication except in the trivial case where $\mathcal{H}$ and $C$ are simultaneously unitarily equivalent to $\mathbb{C}$ and complex conjugation, respectively. Indeed, it is easy to find complex symmetric $2 \times 2$ matrices whose product is not complex symmetric.

Example 8. Hankel operators are $C$-symmetric operators since every Hankel matrix is complex symmetric. For instance, the Carleman operator

$$
(\Gamma f)(x)=\int_{0}^{\infty} \frac{f(y)}{x+y} d y
$$

on $L^{2}(0, \infty)$ is $C$-symmetric since it can be represented as a Hankel matrix with respect to a certain orthonormal basis [40, p. 55].

Example 9. As a simple example, consider the Jordan block $J:=J_{3}(\lambda)$ of order 3 acting on $\mathbb{C}^{3}$. That is,

$$
J=\left(\begin{array}{lll}
\lambda & 1 & 0 \\
0 & \lambda & 1 \\
0 & 0 & \lambda
\end{array}\right) .
$$

The vectors $e_{1}=\frac{1}{\sqrt{2}}(1,0,1), e_{2}=\frac{1}{\sqrt{2}}(i, 0,-i)$, and $e_{3}=(0,1,0)$ are orthonormal and self-conjugate with respect to the symmetry

$$
C\left(z_{1}, z_{2}, z_{3}\right):=\left(\overline{z_{3}}, \overline{z_{2}}, \overline{z_{1}}\right) .
$$

The matrix for $J$ with respect to the basis $\left\{e_{1}, e_{2}, e_{3}\right\}$ is the matrix

$$
\left(\begin{array}{ccc}
\lambda & 0 & \frac{1}{\sqrt{2}} \\
0 & \lambda & \frac{-i}{\sqrt{2}} \\
\frac{1}{\sqrt{2}} & \frac{-i}{\sqrt{2}} & \lambda
\end{array}\right)
$$

which is complex symmetric, as expected. Similar results hold of course for Jordan blocks of higher order.

Example 10. A Toeplitz matrix of finite order $n$ defines a $C$-symmetric operator on $\mathbb{C}^{n}$. Indeed we have $C_{n} T=T^{*} C_{n}$ where $C_{n}$ denotes the involution (8) on $\mathbb{C}^{n}$. Toeplitz operators on $H^{2}$ are in general not $C$-symmetric, although their compressions to coinvariant subspaces for the unilateral shift are (Subsection 4.4).

One of the oldest and most important results about complex symmetric matrices is the following theorem (originating in the work of Takagi [51] and reproved in different contexts at least by Schur, Hua, Siegel and Jacobson; see the comments in [30]). The infinite dimensional proof below is a simple adaptation of Siegel's proof, [49. Lemma 1].

Theorem 1 (Takagi Factorization). Let $T=T^{t}$ be a symmetric matrix representation of a $C$-symmetric operator. There exists a unitary matrix $U$ and a normal and symmetric matrix $N$ (with respect to the same basis), such that

$$
T=U N U^{t} \text {. }
$$

Proof. Note that $T \bar{T}=T T^{*}$ is a self-adjoint matrix, therefore there exists a unitary $U$ and a real symmetric matrix $S$ such that $T \bar{T}=U S U^{*}$. Then note that $\bar{T} T=\bar{U} S U^{t}$ and that the matrix $N=U^{*} T \bar{U}$ is normal $\left(N N^{*}=N^{*} N=S\right)$ and symmetric $\left(N=N^{t}\right)$. Thus $T=U N U^{t}$ as stated. In the case of finite matrices, 
$N$ can be further diagonalized by a real orthogonal matrix $O: N=O D O^{t}$; see 30.

In a similar spirit, we have the following theorem:

Theorem 2. If $T$ is a $C$-symmetric operator, then the antilinear operator $C T$ commutes with the spectral measure of $T^{*} T$. In other words, if $E=E_{T^{*} T}$ denotes the spectral measure of $T^{*} T$, then

$$
C T E(\sigma)=E(\sigma) C T
$$

for every Borel subset $\sigma$ of $[0, \infty)$.

Proof. $C T$ commutes with $T^{*} T$ since $(C T)^{2}=T^{*} T$. Thus $C T$ commutes with $p\left(T^{*} T\right)$ for any polynomial $p(x)$ with real coefficients and hence with each $E(\sigma)$.

Equivalently, one can also say that the antilinear operator $T C$ commutes with the spectral measure of $T T^{*}$.

\subsection{Remarks.}

a. Aiming at a general model for $C$-symmetric operators $T$, we remark that in this case both $\operatorname{Re} T$ and $\operatorname{Im} T$ belong to $C^{\circ}$ and that there is no a priori relation between $\operatorname{Re} T$ and $\operatorname{Im} T$. Thus, the standard process of diagonalizing $\operatorname{Re} T$ on a direct integral of Hilbert spaces and representing $\operatorname{Im} T$ there as an integral operator gives little insight into the structure of $T$.

b. Theorem 2 suggests that a functional model of $T \in C^{\circ}$ based on the diagonalization of $T^{*} T$ is within reach. To be more specific, consider a direct integral decomposition of $T^{*} T=M_{x}$, on which this operator is represented by the multiplication by the real variable $x$. This representation carries its own natural symmetry, $S f(x)=\overline{f(x)}$, which commutes with $M_{x}$ and even with the spectral measure $E$ of this operator. Using Theorem 2 we see that the complex linear operator CTS satisfies

$$
C T S E(\sigma)=E(\sigma) C T S
$$

for any Borel subset $\sigma$ of $[0, \infty)$. Moreover, $C T S$ belongs to the commutant of $T^{*} T$ and there exists a fibre preserving, operator-valued, essentially bounded function $h(x)$ such that

$$
[C T S f](x)=h(x) f(x) .
$$

Furthermore, $h(x)$ satisfies $h(x)^{*} h(x)=x I$ almost everywhere. This yields the following description of $T$ :

$$
T f(x)=C[h \bar{f}](x) .
$$

The structure of the symmetry $C$, on the direct integral representation of the underlying Hilbert space, remains rather abstract. We will give one example below (see Section 4.3) where the computations on this model become explicit.

We plan to resume this subject in a separate article. 
c. The set $C^{\circ}$ is relevant for Siegel's analogue of the homogeneous, hyperbolic geometry of the planar unit disk or upper half-plane. More specifically,

$$
\mathbf{B}(C)=\left\{T \in C^{\circ}:\|T\|<1\right\}
$$

is Siegel's correspondent of the unit ball, and

$$
\mathbf{H}(C)=\left\{T \in C^{\circ}: \operatorname{Im} T>0\right\}
$$

of the upper half-plane; see [49] or [33].

The connection between the homogeneous complex structure of $\mathbf{H}(C)$ and similar matrix realizations of symmetric domains and operator theory was long ago established and exploited by Potapov, Krein, Livsic and their followers; see for instance [27, 28, 41]. Within our framework we mention only that a self-adjoint $C$-symmetric operator $A$ (bounded or not) has a resolvent $R(z)=(A-z)^{-1}$ defined in the upper half-plane, and with values in $\mathbf{H}(C)$ :

$$
C(A-z)^{-1}=(A-z)^{-1} C
$$

and

$$
\frac{1}{2 i}\left[(A-\bar{z})^{-1}-(A-z)^{-1}\right]=(A-\bar{z})^{-1} \frac{\bar{z}-z}{2 i}(A-z)^{-1}>0, \quad \operatorname{Im} z>0 .
$$

The homogeneous structure of $\mathbf{H}(C)$ can lead, as in the cases studied by the above authors, to canonical representations of such resolvent functions. We do not follow this direction here.

\section{Concrete $C$-Symmetric operators}

The aim of this section is to provide a series of (quite distinct) examples of $C$-symmetric operators.

4.1. Normal operators. The building blocks (that is orthogonal summands) of any normal operator are the multiplication operators $M_{z}$ on a Lebesgue space $L^{2}(\mu)$ of a planar, positive Borel measure $\mu$ with compact support. It is clear that complex conjugation $C f=\bar{f}$ is isometric and that $C M_{z}=M_{z}^{*} C$.

Subnormal operators are not in general $C$-symmetric, due to the fact that they tend to have nonzero Fredholm index on some part of their spectrum (see [53]). For instance, the unilateral shift represented as the multiplication operator $M_{z}$ on the Hardy space $H^{2}$ of the disk cannot be $C$-symmetric, as was already clear from Proposition 1. The same conclusion obviously applies to the Bergman shift operator $M_{z}$ with respect to any bounded planar domain.

4.2. Finite rank and compact matrices. Recall that $u \otimes v$ denotes the rankone operator $(u \otimes v) f:=\langle f, v\rangle u$ and that any rank-one operator on $\mathcal{H}$ has such a representation.

Lemma 2. The operator $T=u \otimes v$ satisfies $C T=T^{*} C$ if and only if $T$ is a constant multiple of $u \otimes C u$.

Proof. Indeed, it is easy to see that $C(u \otimes v)=(C u \otimes C v) C$ since

$$
C\langle f, v\rangle u=\langle v, f\rangle C u=\langle C f, C v\rangle C u
$$


for all $f, u, v \in \mathcal{H}$. Now $(u \otimes v)^{*}=v \otimes u$ and hence

$$
C(u \otimes v)=(u \otimes v)^{*} C
$$

if and only if $v \otimes u=C u \otimes C v$.

Passing now to compact operators, it is easy to construct $C$-symmetric ones. For instance, if $u_{n}$ is a sequence of unit vectors in $\mathcal{H}$ and $a_{n}$ is an absolutely summable sequence of scalars, then the operator

$$
T=\sum_{n=1}^{\infty} a_{n}\left(u_{n} \otimes C u_{n}\right)
$$

is bounded and satisfies $C T=T^{*} C$. Under certain circumstances, we can use Theorem 2 to obtain a similar decomposition of a compact $C$-symmetric operator. Consider the following example.

Example 11. If $T$ is a compact $C$-symmetric operator such that $T T^{*}$ is injective and has simple spectrum, then we may write

$$
T T^{*}=\sum_{n=1}^{\infty} c_{n}\left(u_{n} \otimes u_{n}\right)
$$

where the $c_{n}$ are distinct positive constants tending to 0 and the vectors $u_{n}$ form an orthonormal basis of the underlying Hilbert space. By Theorem 2, the onedimensional eigenspaces of $T T^{*}$ are fixed by the antilinear operator $T C$ and hence there exist complex constants $a_{n}$ such that

$$
T C u_{n}=a_{n} u_{n}, \quad n \geq 1 .
$$

Since $T C T C u_{n}=T T^{*} u_{n}=c_{n} u_{n}$, we see that $\left|a_{n}\right|^{2}=c_{n}$. This yields the decomposition

$$
T=\sum_{n=1}^{\infty} a_{n}\left(u_{n} \otimes C u_{n}\right)
$$

of the operator $T$. Convergence is assured by the orthonormality of the vectors $u_{n}$ and by the fact that the coefficients $a_{n}$ tend to 0 .

We leave it to the reader to make the appropriate modifications in the case where $T T^{*}$ does not have simple spectrum.

It is worth mentioning at this point Hamburger's example of a compact operator $K$, with a complete system of root vectors, such that $K^{*}$ does not have a complete system of root vectors [29. Thus, $K$ cannot be similar to a $C$-symmetric operator.

4.3. Volterra operators. Consider the simplest Volterra operator

$$
V f(x)=\int_{0}^{x} f(t) d t
$$

on $L^{2}[0,1]$. The involution $C f(t):=\overline{f(1-t)}$ is a conjugation operator and a straightforward computation shows that $V$ is $C$-symmetric.

In a similar way we can treat more general Volterra type operators. More specifically, let $\mathcal{L}$ be an auxiliary Hilbert space with an antilinear, isometric involution $J$, and let $A:[0,1] \longrightarrow B(\mathcal{L})$ be an essentially bounded, measurable, operator-valued function, with values in $J^{\circ}$ :

$$
A(t) J=J A(t)^{*}, \quad t \in[0,1], \text { a.e. }
$$


On the vector-valued Lebesgue space $\mathcal{H}=L^{2}[0,1] \hat{\otimes} \mathcal{L}$ we define the involution

$$
(C f)(t)=J(f(1-t)), \quad f \in \mathcal{H}, t \in[0,1] .
$$

A straightforward computation shows that the Volterra type operator

$$
V f(t)=\int_{0}^{t} A(t-s) f(s) d s, \quad f \in \mathcal{H}
$$

satisfies the $C$-symmetry relation $C V=V^{*} C$.

To give a numerical example, on $L^{2}[0,1]$, we consider the Abel-Liouville potentials:

$$
\left(J_{\alpha} f\right)(t)=\frac{1}{\Gamma(\alpha)} \int_{0}^{t}(t-s)^{\alpha-1} f(s) d s, \quad \alpha>0 .
$$

They are simultaneously $C$-symmetric with respect to the symmetry $C f(t)=$ $\overline{f(1-t)}$.

Volterra operators $V$ of real type (that is, satisfying $C V=V C$ ) were extensively studied by the Ukrainian school; see [27. It is interesting to note that the canonical models for these real operators involve only the pointwise involution $(C f)(t)=\overline{f(t)}$, but not the argument inversion $(t \mapsto 1-t)$ we had above.

4.4. Compressions of Toeplitz operators. We maintain the notation of Example 1 and freely identity functions in $H^{2}$ with their boundary values on the unit circle. In particular, recall the definitions (2) and (3) of the Hilbert space $\mathcal{H}_{\varphi}$ and conjugation operator $C$, respectively.

For a nonconstant function $u$ belonging to $L^{\infty}$, the Toeplitz operator with symbol $u$ is the operator on $H^{2}$ given by

$$
T_{u} f:=P(u f)
$$

where $P$ denotes the orthogonal projection from $L^{2}$ onto $H^{2}$. It is well known that $T_{u}^{*}=T_{\bar{u}}$ for each $u$ in $L^{\infty}$. For a nonconstant inner function $\varphi$, the compression of $T_{u}$ to $\mathcal{H}_{\varphi}$ is the operator

$$
\mathrm{T}_{u}:=P_{\varphi} T_{u} P_{\varphi}
$$

where $P_{\varphi}$ denotes the orthogonal projection from $H^{2}$ onto $\mathcal{H}_{\varphi}$. These operators are simultaneously $C$-symmetric.

Proposition 3. If $\varphi$ is a nonconstant inner function, then $\left(\mathcal{H}_{\varphi}, \mathrm{T}_{u}, C\right)$ is a $C$ symmetric triple for each $u$ belonging to $L^{\infty}$.

Proof. If $f$ and $g$ belong to $\mathcal{H}_{\varphi}$, then

$$
\begin{aligned}
\left\langle C \mathrm{~T}_{u} f, g\right\rangle & =\left\langle C g, \mathrm{~T}_{u} f\right\rangle=\left\langle C g, P_{\varphi} T_{u} P_{\varphi} f\right\rangle \\
& =\left\langle P_{\varphi} C g, T_{u} f\right\rangle=\langle C g, P(u f)\rangle \\
& =\langle P C g, u f\rangle=\langle C g, u f\rangle \\
& =\langle\overline{g z} \varphi, u f\rangle=\langle\overline{f z} \varphi, u g\rangle \\
& =\langle C f, u g\rangle=\left\langle P P_{\varphi} C f, u g\right\rangle \\
& =\left\langle P_{\varphi} C f, T_{u} g\right\rangle=\left\langle C f, P_{\varphi} T_{u} P_{\varphi} g\right\rangle \\
& =\left\langle C f, \mathrm{~T}_{u} g\right\rangle=\left\langle\mathrm{T}_{u}^{*} C f, g\right\rangle .
\end{aligned}
$$

Hence $C \mathrm{~T}_{u}=\mathrm{T}_{u}^{*} C$ as desired. 
In particular, the compression of the unilateral shift to $\mathcal{H}_{\varphi}$ (known as a standard model operator or Jordan operator) is $C$-symmetric (see also [35, Lemma 9.2]. We will examine this operator in more detail in Section 6.

If $h$ belongs to $H^{\infty}$, then several well-known classical results (see, for instance, [8, 39]) follow from the fact that the compression $\mathrm{T}_{h}$ of the multiplication operator $T_{h}$ (on $H^{2}$ ) is $C$-symmetric. For instance, the correspondence between portions of the spectra of a $C$-symmetric operator and its adjoint discussed after Proposition 1 apply immediately to the operators $\mathrm{T}_{h}$ and $\mathrm{T}_{h}^{*}$. This is in stark contrast to the (uncompressed) operators $T_{h}$ and $T_{h}^{*}$ on $H^{2}$. In general, the spectra of $T_{h}$ and $T_{h}^{*}$ are structurally quite different. For example, if $h$ is nonconstant, then $T_{h}$ has empty point spectrum whereas the point spectrum of $T_{h}^{*}$ contains $\overline{h(\mathbb{D})}$.

\section{Extensions of $C$-SYMmetric OpERATORS}

Compared to the dilation and extension theory in spaces with an indefinite metric (see, for instance, 2]), the analogous results for $C$-symmetric operators are much simpler. We consider in this section only two illustrative situations.

Let $S: \mathcal{D} \longrightarrow \mathcal{H}$ be a densely defined, closed graph symmetric operator. Recall von-Neumann's criterion for the existence of a self-adjoint extension of $S$ : If there exists an antilinear involution $C: \mathcal{D} \longrightarrow \mathcal{D}$ such that $C S=S C$, then the defect numbers of $S$ are equal, hence at least one self-adjoint extension of $S$ exists; see [37) and [43, 45].

The special case of an isometric involution $C$, actually considered by von Neumann ([37], p. 101) is interesting for us, because among all self-adjoint extensions, only part of them turn out to be $C$-symmetric. These operators, and extensions, were called real by von Neumann.

Proposition 4. Let $S: \mathcal{D} \longrightarrow \mathcal{H}$ be a closed graph, densely defined symmetric operator and assume that there exists an antilinear, isometric involution $C: \mathcal{H} \longrightarrow$ $\mathcal{H}$ mapping $\mathcal{D}$ into itself and satisfying the symmetry relation $S C=C S$. Then the $C$-symmetric self-adjoint extensions $A$ (i.e. $A=C A C$ ) of $S$ are parametrized by all isometric maps $V: \operatorname{Ker}\left(S^{*}-i\right) \longrightarrow \operatorname{Ker}\left(S^{*}+i\right)$ satisfying $V^{*} C=C V$.

Proof. Indeed, the involution $C$ maps the defect space $\operatorname{Ker}\left(S^{*}-i\right)$ into $\operatorname{Ker}\left(S^{*}+i\right)$. Let A be a $C$-symmetric self-adjoint extension of $S$ corresponding to the isometry $V: \operatorname{Ker}\left(S^{*}-i\right) \longrightarrow \operatorname{Ker}\left(S^{*}+i\right)$; see for instance [43. Then the graph of $A$ consists of $\operatorname{Graph}(S) \oplus\left\{(f, V f): f \in \operatorname{Ker}\left(S^{*}-i\right)\right\}$, and $A f=i f, A V f=-i V f$. Since the domain of $A$ is invariant under $C$ we infer $(C V f, C f)=(g, V g)$ for some $g \in \operatorname{Ker}\left(S^{*}-i\right)$. In other words, $V^{*} C=C V$.

The case of defect indices $(1,1)$ is simple, for any self-adjoint extension of $S$ is $C$-symmetric, due to the observation $C\left(e^{i t}\right)=e^{-i t} C$ for any real parameter $t$. For higher defect indices, however, not all self-adjoint extensions are $C$-symmetric.

We remark that the Ukrainian school (I.M. Glazman in particular) investigated the change acquired in the spectrum of an unbounded $C$-symmetric operator $S$ (i.e. $S \subset C S^{*} C$ ) when completed to one of its $C$-self-adjoint extensions $\tilde{S}$ (i.e. $S \subset \tilde{S}$ and $\tilde{S}=C \tilde{S}^{*} C$ ). Interestingly enough, these observations apply to SturmLiouville operators of the form $-u^{\prime \prime}+q(x) u, u \in L^{2}(-\infty, \infty)$, where $q(x)$ is a nonreal potential [24, Sect. 23-34]. 
We turn now to a $C$-symmetric contractive operator $T \in B(\mathcal{H})$. The defect spaces of $T$ are

$$
\mathcal{D}_{+}=\operatorname{Ran}\left(I-T^{*} T\right)^{1 / 2}, \quad \mathcal{D}_{-}=\operatorname{Ran}\left(I-T T^{*}\right)^{1 / 2},
$$

where Ran $A$ denotes the norm closure of the range of the operator $A$. If $C T=T^{*} C$, then $C T^{*} T=T T^{*} C$ and hence

$$
C\left(I-T^{*} T\right)^{1 / 2}=\left(I-T T^{*}\right)^{1 / 2} C .
$$

In particular, this shows that

$$
C: \mathcal{D}_{+} \longrightarrow \mathcal{D}_{-}
$$

is an isometric antilinear map.

Thus a $C$-symmetric contraction must have equal dimensional defect spaces. The Sz.-Nagy minimal unitary dilation $U$ of $T$ can be constructed as an infinite matrix (see [16]) as recalled below.

Let

$$
\mathcal{K}=\ldots \oplus \mathcal{D}_{-} \oplus \mathcal{D}_{-} \oplus \mathcal{H} \oplus \mathcal{D}_{+} \oplus \mathcal{D}_{+} \ldots
$$

be a direct sum Hilbert space with $\mathcal{H}$ on the 0 -th position (marked below in bold face characters). Let $U: \mathcal{K} \longrightarrow \mathcal{K}$ be the operator explicitly defined by

$$
\begin{gathered}
U\left(\ldots, x_{-2}, x_{-1}, \mathbf{x}_{\mathbf{0}}, x_{1}, x_{2}, \ldots\right) \\
=\left(\ldots, x_{-3}, x_{-2},\left(\mathbf{I}-\mathbf{T} \mathbf{T}^{*}\right)^{\mathbf{1 / 2}} \mathbf{x}_{-\mathbf{1}}+\mathbf{T} \mathbf{x}_{\mathbf{0}},-T^{*} x_{-1}+\left(I-T^{*} T\right)^{1 / 2} x_{0}, x_{1}, \ldots\right) .
\end{gathered}
$$

It is easy to prove that $U$ is a unitary operator which dilates $T$, in the sense that $\left\langle U^{n} x_{0}, x_{0}\right\rangle=\left\langle T^{n} x_{0}, x_{0}\right\rangle, n \in \mathbb{N}$, where $x_{0}$ is a vector supported by the 0 -th position.

We define the isometric antilinear involution $\tilde{C}: \mathcal{K} \longrightarrow \mathcal{K}$ by the formula

$$
\begin{gathered}
\tilde{C}\left(\ldots, x_{-2}, x_{-1}, \mathbf{x}_{\mathbf{0}}, x_{1}, x_{2}, \ldots\right) \\
=\left(\ldots, C x_{2}, C x_{1}, \mathbf{C x}_{\mathbf{0}}, C x_{-1}, C x_{-2}, \ldots\right) .
\end{gathered}
$$

A straightforward computation shows that $U C U=C$ and hence $U$ is $C$-symmetric. In conclusion we have proved the following result.

Theorem 3. Let $T$ be a $C$-symmetric contraction. The map $C$ extends to an antilinear, isometric involution $\tilde{C}$ on the space of the unitary dilation $U$ of $T$, such that $\tilde{C} U=U^{*} \tilde{C}$.

As an almost tautological example we consider the following typical analysis of a self-adjoint extension of defect indices $(1,1)$.

Example 12. Let $s_{0}, s_{1}, \ldots$ be an indeterminate moment sequence of a probability measure on the line. Let $\mathcal{H}$ be the completion of the space of polynomials $\mathbb{C}[x]$ in the norm given by the associated positive definite Hankel matrix

$$
\left\|\sum_{k=0}^{n} c_{k} x^{k}\right\|^{2}=\sum_{k, l=0}^{n} s_{k+l} c_{k} \overline{c_{l}} .
$$

Let $P_{k}(x)$ be the associated orthogonal polynomials, normalized by the condition $\left\|P_{k}\right\|=1$ and the leading term of each $P_{k}$ is positive. In this way $\mathcal{H}$ can be identified with $l^{2}(\mathbb{N})$. These orthogonal polynomials have real coefficients, hence they are invariant under the involution $(C q)(x)=\overline{q(x)}$ for $x$ in $\mathbb{R}$. 
The unbounded operator of multiplication by the variable $x$ can be represented by a Jacobi matrix $J$, formally symmetric in the norm of $\mathcal{H}$. By considering $J$ on its maximal domain of definition $\mathcal{D}$ we obtain a closed graph, symmetric operator with defect indices $(1,1)$ (due to the fact the problem is indeterminate). For all details see for instance [1, 28.

Obviously, the $C$-symmetry relation $C J=J^{*} C=J C$ holds on $\mathcal{D}$. The selfadjoint extensions of $J$ are parametrized by a complex number $\alpha$ of modulus one and can explicitly be given as follows. Fix an arbitrary nonreal complex number $\lambda$ and consider the vector $\Pi_{\lambda}=\left(P_{0}(\lambda), P_{1}(\lambda), \ldots\right) \in \operatorname{Ker}\left(J^{*}-\lambda\right)$. This vector belongs to $l^{2}(\mathbb{N})$ by the indeterminate nature of the moment sequence. Define $P_{\lambda}=\Pi_{\lambda} /\left\|\Pi_{\lambda}\right\|$. Then the rank-one operator $P_{\lambda} \otimes C P_{\lambda}=\left\langle\cdot, P_{\lambda}\right\rangle C P_{\lambda}$ satisfies

$$
\begin{aligned}
{\left[P_{\lambda} \otimes C P_{\lambda}\right]\left(P_{0}(\lambda), P_{1}(\lambda), \ldots\right) } & =C\left(P_{0}(\lambda), P_{1}(\lambda), \ldots\right) \\
& =\frac{\left(P_{0}(\lambda), P_{1}(\lambda), \ldots\right)}{}=\left(P_{0}(\bar{\lambda}), P_{1}(\bar{\lambda}), \ldots\right),
\end{aligned}
$$

and thus it maps the defect space $\operatorname{Ker}\left(J^{*}-\lambda\right)$ isometrically onto $\operatorname{Ker}\left(J^{*}-\bar{\lambda}\right)$.

Thus all self-adjoint extensions of the Jacobi matrix $J$ can be described, on the enlarged domain of definition $\mathcal{D}+\mathbb{C} \Pi_{\lambda}$, as

$$
S_{\alpha}=J+\alpha\left(P_{\lambda} \otimes C P_{\lambda}\right),
$$

for $|\alpha|=1$. A direct computation, or the proposition above, shows that the family $S_{\alpha}$ is simultaneously $C$-symmetric.

\section{Clark perturbations}

In this section we examine the compressions of the unilateral shift onto its coinvariant subspaces $\mathcal{H}_{\varphi}$ from the viewpoint of $C$-symmetry. Maintaining the conventions and notation of Examples 1 and 3 and Subsection 4.4 we show here that the rank-one unitary perturbations of the compressed unilateral shift considered by Clark [11] are jointly $C$-symmetric with respect to the symmetry (31). Indeed, we consider a slight generalization at little extra expense.

6.1. Simultaneous $C$-symmetry. For $\lambda$ in the unit disk define

$$
b_{\lambda}(z):=\frac{z-\lambda}{1-\bar{\lambda} z}
$$

and consider the operator

$$
S_{\lambda}:=P_{\varphi} T_{b_{\lambda}} P_{\varphi}
$$

on $\mathcal{H}_{\varphi}$. Hence $S_{\lambda}$ is simply the compression to $\mathcal{H}_{\varphi}$ of the multiplication operator (on $H^{2}$ ) with symbol $b_{\lambda}$. The case $\lambda=0$ corresponds to the compression of the unilateral shift. Proposition 3 tells us that the operators $S_{\lambda}$ are jointly $C$-symmetric.

The following lemma is a generalization of Clark's initial observation and is phrased in terms of the conjugation operator $C$. Recall the formulas (4) and (5) for the functions $K_{\lambda}$ and $Q_{\lambda}$ described in Example 3 ,

Lemma 3. For each $\lambda$ in $\mathbb{D}$, the following statements hold:

(a) $S_{\lambda} f=b_{\lambda} f$ if and only if $f$ is orthogonal to $Q_{\lambda}$.

(b) $S_{\lambda}^{*} f=f / b_{\lambda}$ if and only if $f$ is orthogonal to $K_{\lambda}$. 
Proof. Clearly, $S_{\lambda}^{*} f=f / b_{\lambda}$ if and only if $f / b_{\lambda}$ belongs to $H^{2}$. This happens if and only if $f(\lambda)=0$, or equivalently, if and only if $\left\langle f, K_{\lambda}\right\rangle=0$. By the preceding, $S_{\lambda}^{*} C f=(C f) / b_{\lambda}$ if and only if $\left\langle C f, K_{\lambda}\right\rangle=0$, or equivalently, if and only if $f$ is orthogonal to $Q_{\lambda}=C K_{\lambda}$. Since $C S_{\lambda}=S_{\lambda}^{*} C$, this implies that $S_{\lambda} f=C\left[C f / b_{\lambda}\right]=$ $b_{\lambda} f$ if and only if $f$ is orthogonal to $Q_{\lambda}$.

At this point it is convenient to introduce the normalized kernel functions $k_{\lambda}$ and $q_{\lambda}$ defined by

$$
k_{\lambda}:=\frac{K_{\lambda}}{\left\|K_{\lambda}\right\|}, \quad q_{\lambda}:=\frac{Q_{\lambda}}{\left\|Q_{\lambda}\right\|} .
$$

For each $\alpha$ of unit modulus, the operator

$$
U_{\lambda, \alpha}:=S_{\lambda}\left[I-\left(q_{\lambda} \otimes q_{\lambda}\right)\right]+\alpha\left(k_{\lambda} \otimes q_{\lambda}\right)
$$

is unitary by the preceding proposition. Moreover, it is a rank-one perturbation of $S_{\lambda}$ since

$$
U_{\lambda, \alpha}=S_{\lambda}+(\alpha+\varphi(\lambda))\left(k_{\lambda} \otimes C k_{\lambda}\right)
$$

as a straightforward computation shows. The proof that the $U_{\lambda, \alpha}$ are the only rankone unitary perturbations of $S_{\lambda}$ is a straightforward generalization of the original proof [1] and left to the reader.

Our interest in these perturbations stems from the fact that they are jointly $C$-symmetric. The following proposition follows immediately from Proposition 3 and Lemma 2 ,

Proposition 5. If $\varphi$ is a nonconstant inner function, then the operators $U_{\lambda, \alpha}$ are jointly $C$-symmetric. That is, $\left(\mathcal{H}_{\varphi}, U_{\lambda, \alpha}, C\right)$ is a $C$-symmetric triple for each $|\lambda|<1$ and each $|\alpha|=1$.

By (13) we have

$$
U_{\lambda, \alpha} f=S_{\lambda} f+(\alpha+\varphi(\lambda)) \overline{C f(\lambda)} \frac{1-|\lambda|^{2}}{1-|\varphi(\lambda)|^{2}} K_{\lambda}
$$

for each $f$ in $\mathcal{H}_{\varphi}$. Thus the antilinear operator $C$ plays a hidden role in the structure of the $U_{\lambda, \alpha}$, so the rank-one perturbing operator involves the twisted point evaluation $f \mapsto \overline{C f(\lambda)}$.

6.2. $C$-real bases. Under certain circumstances we can explicitly furnish a selfconjugate orthonormal basis $e_{n}$ for $\mathcal{H}_{\varphi}$. Although the existence of such a basis is guaranteed by Lemma1, we are interested here in producing them for the purpose of computing the corresponding matrix representation of the compressed shift operator $S:=S_{0}$.

Let $\alpha$ be a unimodular constant and consider the unitary operator $U_{\alpha}:=U_{0, \alpha}$. It was shown in [11, Lemma 3.1] that a complex number $\zeta$ is an eigenvalue of $U_{\alpha}$ if and only if $\varphi$ has a finite angular derivative $\varphi^{\prime}(\zeta)$ at $\zeta$ (note that $|\zeta|=1$ since $U_{\alpha}$ is unitary) and $\varphi(\zeta)=\beta$ where the unimodular constant $\beta$ is defined by

$$
\beta:=\frac{\alpha+\varphi(0)}{1+\overline{\varphi(0)} \alpha} .
$$

The corresponding unit eigenvector will be $k_{\zeta}$, where the definition (11) of $k_{\zeta}$ is extended to include unimodular $\zeta$ in the obvious way. Although the finiteness of 
the angular derivative is not explicit in [11, it is easily seen to be equivalent to the condition above (see [4, p. 367] or [3]).

Let $\zeta_{n}$ denote an enumeration of the (at most countably many) eigenvalues of $U_{\alpha}$. Clark showed that if the operator $U_{\alpha}$ has pure point spectrum, then the corresponding eigenvectors $k_{n}:=k_{\zeta_{n}}$ of $U_{\alpha}$ form an orthonormal basis for $\mathcal{H}_{\varphi}$. This occurs [11, Theorem 7.1] if the set of points on the unit circle at which $\varphi$ does not have a finite angular derivative is countable. For example, the eigenvectors of $U_{\alpha}$ span $\mathcal{H}_{\varphi}$ if $\varphi$ is a Blaschke product whose zeros cluster only on a countable set or if $\varphi$ is a singular inner function such that the closure of the support of the associated singular measure is countable. In such cases the modulus $\left|\varphi^{\prime}\left(\zeta_{n}\right)\right|$ of the angular derivative of $\varphi$ at $\zeta_{n}$ is finite and equals $\left\|K_{n}\right\|^{2}$ where $K_{n}:=K_{\zeta_{n}}$.

Suppose now that $\varphi$ is an inner function and $|\alpha|=1$ such that the corresponding operator $U_{\alpha}$ has pure point spectrum $\left\{\zeta_{1}, \zeta_{2}, \ldots\right\}$, and fix $t_{n}$ for $n=0,1,2, \ldots$ such that $e^{i t_{0}}=\beta$ and $\zeta_{n}=e^{i t_{n}}$ for each $n \geq 1$. The preceding discussion tells us that the functions $k_{n}$ form an orthonormal basis for $\mathcal{H}_{\varphi}$. Multiplying the $k_{n}$ by suitable unimodular constants yields a self-conjugate orthonormal basis $e_{n}$ defined by

$$
e_{n}:=e^{\frac{i}{2}\left(t_{0}-t_{n}\right)} k_{n}
$$

with respect to which each $f$ in $\mathcal{H}_{\varphi}$ enjoys the expansion

$$
f(z)=\sum_{n=1}^{\infty} \frac{e^{\frac{i}{2}\left(t_{n}-t_{0}\right)}}{\sqrt{\left|\varphi^{\prime}\left(\zeta_{n}\right)\right|}} f\left(\zeta_{n}\right) e_{n}(z) .
$$

Whence we obtain the inner product formula

$$
\langle f, g\rangle=\sum_{n=1}^{\infty} \frac{f\left(\zeta_{n}\right) \overline{g\left(\zeta_{n}\right)}}{\left|\varphi^{\prime}\left(\zeta_{n}\right)\right|}
$$

for $f, g$ in $\mathcal{H}_{\varphi}$. Since $C$ is simply the complex conjugation with respect to the basis $e_{n}$, we see that

$$
\Phi f:=\left(\frac{e^{\frac{i}{2}\left(t_{n}-t_{0}\right)}}{\sqrt{\left|\varphi^{\prime}\left(\zeta_{n}\right)\right|}} f\left(\zeta_{n}\right)\right)_{n=1}^{\infty}
$$

is an isometric isomorphism of $\mathcal{H}_{\varphi}$ onto a certain weighted $l^{2}$ space such that

$$
\Phi(C f)=\overline{\Phi f}, \quad f \in \mathcal{H}_{\varphi} .
$$

Moreover, if the sequence $w_{n} / \sqrt{\left|\varphi^{\prime}\left(\zeta_{n}\right)\right|}$ is square-summable, then there exists a function $f$ in $\mathcal{H}_{\varphi}$ whose nontangential limiting values at the points $\zeta_{n}$ interpolate the values $w_{n}$.

6.3. Symmetric Jordan matrices. We now explicitly compute the matrix representation of the compressed shift operator $S$ with respect to the basis $e_{n}$. In particular, we will see that the matrix $\left(\left\langle S e_{n}, e_{m}\right\rangle\right)_{n, m=1}^{\infty}$ is complex symmetric (as expected) and, moreover, that the entries are related to the eigenvalues $\zeta_{n}$ in a simple way. By (14) we have

$$
S e_{n}=\zeta_{n} e_{n}-\overline{e_{n}(0)} \frac{\alpha+\varphi(0)}{1-|\varphi(0)|^{2}} K_{0}
$$


where we used the fact that $e_{n}$ are self-conjugate eigenvectors for $U_{\alpha}$. Thus

$$
\begin{aligned}
\left\langle S e_{n}, e_{m}\right\rangle & =\zeta_{n}\left\langle e_{n}, e_{m}\right\rangle+\overline{e_{n}(0)} \frac{\alpha+\varphi(0)}{1-|\varphi(0)|^{2}}\left\langle K_{0}, e_{m}\right\rangle \\
& =\zeta_{n} \delta_{n m}+\overline{e_{n}(0) e_{m}(0)} \frac{\alpha+\varphi(0)}{1-|\varphi(0)|^{2}} \\
& =\zeta_{n} \delta_{n m}+\frac{e^{\frac{i}{2}\left(t_{n}-t_{0}\right)+\left(t_{m}-t_{0}\right)}}{\left\|K_{n}\right\|\left\|K_{m}\right\|}(1-\beta \overline{\varphi(0)})^{2} \frac{\alpha+\varphi(0)}{\left(1-|\varphi(0)|^{2}\right)} \\
& =\zeta_{n} \delta_{n m}+\frac{\zeta_{n}^{\frac{1}{2}} \zeta_{m}^{\frac{1}{2}}}{\left|\varphi^{\prime}\left(\zeta_{n}\right)\right|^{\frac{1}{2}}\left|\varphi^{\prime}\left(\zeta_{m}\right)\right|^{\frac{1}{2}}} \frac{(1-\beta \overline{\varphi(0)})^{2}(\alpha+\varphi(0))}{\left(1-|\varphi(0)|^{2}\right)}
\end{aligned}
$$

Here $\delta_{n m}$ denotes the Kronecker $\delta$-function and the square roots of $\zeta_{n}$ and $\zeta_{m}$ are defined in the obvious way. From this calculation we observe that $\left\langle S e_{n}, e_{m}\right\rangle=$ $\left\langle S e_{m}, e_{n}\right\rangle$ for all $n, m$ and hence the matrix for $S$ with respect to the basis $e_{n}$ is complex symmetric. Moreover, the matrix representations of the unitary operator $U_{\alpha}$ and the perturbing operator are evident in (16). Summing up, we have proved the following result.

Theorem 4. Let $\varphi$ be a nonconstant inner function (on the unit disk) and let $S$ be the standard Jordan operator (the compressed shift) on the model space $\mathcal{H}_{\varphi}:=$ $H^{2} \ominus \varphi H^{2}$. If the rank-one unitary perturbation $U_{\alpha}$ of $S$ has pure point spectrum $\left\{\zeta_{n} ; n \geq 1\right\}$, then there exists an orthonormal basis $e_{n}$ of eigenvectors for $U_{\alpha}$ such that

(1) $C e_{n}=e_{n}$ for all $n \geq 1$ where $C f:=\overline{f z} \varphi$.

(2) The matrix of $S$ with respect to the basis $e_{n}$ is complex symmetric and has the form (16).

In the case $\varphi(0)=0$ our computation reduces to

$$
\left\langle S e_{n}, e_{m}\right\rangle=\zeta_{n} \delta_{n m}+\alpha \frac{\zeta_{n}^{\frac{1}{2}} \zeta_{m}^{\frac{1}{2}}}{\left|\varphi^{\prime}\left(\zeta_{n}\right)\right|^{\frac{1}{2}} \mid \varphi^{\prime}\left(\zeta_{m}\right)^{\frac{1}{2}}} .
$$

\section{7. $C$-SymmetriC STRUCTURE OF MODEL SPACES}

7.1. Inner-outer factorization. We relate the $C$-symmetry $C f=\overline{f z} \varphi$ on the model space $\mathcal{H}_{\varphi}:=H^{2} \ominus \varphi H^{2}$ to the inner-outer factorization of functions $f$ in $\mathcal{H}_{\varphi}$. In terms of boundary functions $\mathcal{H}_{\varphi}$ we have

$$
\mathcal{H}_{\varphi}=H^{2} \cap \varphi \overline{z H^{2}}
$$

and hence the following easy lemma.

Lemma 4. Two functions $f$ and $g$ in $H^{2}$ satisfy $g=\overline{f z} \varphi$ a.e. on $\partial \mathbb{D}$ if and only if $f$ and $g$ belong to $\mathcal{H}_{\varphi}$ and $C f=g$.

Suppose now that $f$ belongs to $\mathcal{H}_{\varphi}$. Since the functions $f$ and $C f$ have the same modulus a.e. on $\partial \mathbb{D}$, they share the same outer factor, say $F$. We may therefore write $f=I_{f} F$ and $C f=I_{C f} F$ where $I_{f}$ and $I_{C f}$ denote the inner factors of $f$ and $C f$, respectively. Moreover,

$$
I_{f} I_{C f} F=\overline{F z} \varphi
$$

a.e. on $\partial \mathbb{D}$. This shows that $F$ belongs to $\mathcal{H}_{\varphi}$ and satisfies $C F=I_{f} I_{C f} F$. Moreover, the inner function $I_{f} I_{C f}$ depends only upon $F$ and $\varphi$ and not on the particular 
pair of conjugate functions $f, C f$ with common outer factor $F$. We call the inner function $I_{f} I_{C f}$ the associated inner function of $F$ (with respect to $\varphi$ ) and denote it $\mathcal{I}_{F}$. The functions $f=I_{f} F$ in $\mathcal{H}_{\varphi}$ with outer factor $F$ are precisely those functions whose inner factors $I_{f}$ divide $\mathcal{I}_{F}$. This yields the following lemma.

Lemma 5. For any outer function $F$ in $\mathcal{H}_{\varphi}$ there exists a unique inner function $\mathcal{I}_{F}$ such that $\mathcal{I}_{F} F=\bar{F} \bar{z} \varphi$ a.e. on $\partial \mathbb{D}$. If $I$ is an inner function, then IF belongs to $\mathcal{H}_{\varphi}$ if and only if I divides $\mathcal{I}_{F}$.

Example 13. Fix a nonconstant inner function $\varphi$ and consider the kernel functions $K_{\lambda}$ and $Q_{\lambda}=C K_{\lambda}$ defined by (4) and (5). The associated inner function for $K_{\lambda}$ is the inner factor

$$
\mathcal{I}_{K_{\lambda}}=\frac{b_{\varphi(\lambda)}(\varphi(z))}{b_{\lambda}(z)}
$$

of $Q_{\lambda}$. Here $b_{\varphi(\lambda)}$ and $b_{\lambda}$ are Möbius transformations defined by (10).

To sum up, a function $f$ belonging to $\mathcal{H}_{\varphi}$ possesses the representations

(1) $f=I_{f} F$ where $F$ is outer, $I_{f}$ is the inner factor of $f$, and $I_{f} \mid \mathcal{I}_{F}$.

(2) $f=f_{1}+i f_{2}$ where $C f_{k}=f_{k}$ for $k=1,2$.

In light of the fact that $|f|^{2}=\left|f_{1}\right|^{2}+\left|f_{2}\right|^{2}$ a.e. on $\partial \mathbb{D}$, it is not difficult to pass from one representation to the other.

7.2. Finite-dimensional model spaces. We consider below the structure of the $N$-dimensional model space $\mathcal{H}_{\varphi}$ corresponding to a finite Blaschke product

$$
\varphi(z)=\prod_{n=1}^{N} \frac{z-\lambda_{n}}{1-\overline{\lambda_{n}} z}
$$

with $N$ (not necessarily distinct) zeroes $\lambda_{n}$. In particular, we make extensive use of the bilinear form $[\cdot, \cdot]$ arising from the conjugation operator $C f=\overline{f z} \varphi$ on $\mathcal{H}_{\varphi}$.

Select any $w$ in the closed unit disk such that the equation $\varphi(z)=w$ has $N$ distinct solutions $z_{1}, \ldots, z_{N}$. The $N$ functions $Q_{n}(z):=Q_{z_{n}}(z)$ defined by (5) are pairwise orthogonal with respect to the bilinear form $[\cdot, \cdot]$ :

$$
\left[Q_{n}, Q_{m}\right]= \begin{cases}\varphi^{\prime}\left(z_{n}\right), & n=m \\ 0, & n \neq m,\end{cases}
$$

and are linearly independent since $\varphi^{\prime}\left(z_{n}\right) \neq 0$ for all $n$. Therefore, the $Q_{n}$ form a basis for $\mathcal{H}_{\varphi}$.

For any $f$ in $\mathcal{H}_{\varphi}$ the well-known interpolation formula

$$
f(z)=\sum_{n=1}^{N} \frac{f\left(z_{n}\right)}{\varphi^{\prime}\left(z_{n}\right)} Q_{n}(z)
$$

follows immediately from (17). The space $\mathcal{H}_{\varphi}$ is essentially a weighted version of $\mathbb{C}^{n}$ twisted by $C$ :

$$
\langle f, g\rangle=\sum_{n=1}^{N} \frac{f\left(z_{n}\right) C g\left(z_{n}\right)}{\varphi^{\prime}\left(z_{n}\right)} .
$$

With respect to the bilinear form $[\cdot, \cdot]$ we have

$$
[f, g]=\sum_{n=1}^{N} \frac{f\left(z_{n}\right) g\left(z_{n}\right)}{\varphi^{\prime}\left(z_{n}\right)} .
$$


We can make these computations more explicit. Each function $f$ belonging to $\mathcal{H}_{\varphi}$ is of the form $f=F / R$ where $F$ is a polynomial of degree $\leq N-1$ and

$$
R(z)=\left(1-\overline{\lambda_{1}} z\right) \cdots\left(1-\overline{\lambda_{N}} z\right) .
$$

The conjugation operator on $\mathcal{H}_{\varphi}$ is given by the formula

$$
C(F / R)=F^{\#} / R
$$

where the polynomial $F^{\#}$ is defined by

$$
F^{\#}(z)=z^{N-1} \overline{F(1 / \bar{z})} .
$$

Observe that $\varphi=P / R$ where $P=z^{N} \overline{R(1 / \bar{z})}$ and then choose any $w$ in the closed unit disk such that the equation

$$
P(z)-w R(z)=0
$$

has $N$ distinct solutions $z_{1}, \ldots, z_{N}$. In other words, select $w$ so that the level set $\varphi^{-1}(\{w\})$ contains $N$ distinct points. (18),

Letting $f=F / R$ and $g=G / R$ denote arbitrary functions in $\mathcal{H}_{\varphi}$ we have by

$$
\begin{aligned}
& {[f, g]=\sum_{n=1}^{N} \frac{F\left(z_{n}\right) G\left(z_{n}\right)}{\Delta\left(z_{n}\right)},} \\
& \langle f, g\rangle=\sum_{n=1}^{N} \frac{F\left(z_{n}\right) G^{\#}\left(z_{n}\right)}{\Delta\left(z_{n}\right)}
\end{aligned}
$$

where the polynomial $\Delta$ is defined by

$$
\Delta=R P^{\prime}-P R^{\prime} .
$$

Note that in the above formulas, the products $[f, g]$ and $\langle f, g\rangle$ are intrinsic, while the right-hand sides depend on the chosen fibre of the function $\varphi$.

7.3. Interpolation theory. This is again a classical and well charted territory which we touch upon only briefly. In this framework $C$-symmetry is a unifying concept and transparent formalism.

Let $\lambda_{n}$ be a sequence of distinct points in the unit disk and define

$$
\varphi(z)=\prod_{n=1}^{\infty} \frac{\left|\lambda_{n}\right|}{\lambda_{n}} \frac{\lambda_{n}-z}{1-\overline{\lambda_{n}} z} .
$$

Since the $\lambda_{n}$ are distinct, it follows that $\varphi^{\prime}\left(\lambda_{n}\right) \neq 0$.

Consider the unit vectors $e_{n}$ defined by

$$
\begin{aligned}
e_{n} & =\frac{Q_{n}}{\left\|Q_{n}\right\|} \\
& =\left(1-\left|\lambda_{n}\right|^{2}\right)^{\frac{1}{2}} Q_{n}
\end{aligned}
$$

where $Q_{n}:=Q_{\lambda_{n}}$ is defined by (5). The $e_{n}$ are orthogonal with respect to the bilinear form $[\cdot, \cdot]$ introduced in Subsection 2.3 since

$$
\left[e_{n}, e_{m}\right]= \begin{cases}\left(1-\left|\lambda_{n}\right|^{2}\right) \varphi^{\prime}\left(\lambda_{n}\right), & n=m, \\ 0, & n \neq m .\end{cases}
$$


We are led therefore to the formal Fourier-type expansion

$$
f=\sum_{n=1}^{\infty} \frac{\left[f, e_{n}\right]}{\left[e_{n}, e_{n}\right]} e_{n}
$$

whose convergent behavior (for all $f$ in $\mathcal{H}_{\varphi}$ ) is naturally linked to the uniform boundedness from below of $\left|\left[e_{n}, e_{n}\right]\right|$. In essence, this is Carleson's famous interpolation theorem.

Recall that a sequence $\lambda_{n}$ in the unit disk is called uniformly separated if there exists a $\delta>0$ such that the Carleson condition

$$
\prod_{k \neq n}\left|\frac{\lambda_{k}-\lambda_{n}}{1-\overline{\lambda_{k}} \lambda_{n}}\right| \geq \delta
$$

holds for every $n$. Thus, according to our computations, this is equivalent to asserting that

$$
\left|\left[e_{n}, e_{n}\right]\right| \geq \delta
$$

for all $n$. In other words, the unit vectors $e_{n}$ are not "asymptotically isotropic" with respect to the bilinear form $[\cdot, \cdot]$.

Carleson's interpolation theorem for $H^{2}$ asserts that the operator

$$
T f:=f\left(\lambda_{n}\right) \sqrt{1-\left|\lambda_{n}\right|^{2}}
$$

maps $\mathcal{H}_{\varphi}$ onto $l^{2}$ if and only if the sequence $\lambda_{n}$ is uniformly separated. Since $T f=\left[f, e_{n}\right]$ for all $n$, the interpolation theorem implies the following result.

Theorem 5. Let $\lambda_{n}$ be a sequence of distinct points in the unit disk, let $\varphi$ be the associated Blaschke product, and let $e_{n}$ be the normalized evaluation elements of the model space $\mathcal{H}_{\varphi}:=H^{2} \ominus \varphi H^{2}$ :

$$
\left[f, e_{n}\right]=f\left(\lambda_{n}\right) \sqrt{1-\left|\lambda_{n}\right|^{2}} .
$$

The $[\cdot, \cdot]$-orthogonal series

$$
f=\sum_{n=1}^{\infty} \frac{\left[f, e_{n}\right]}{\left[e_{n}, e_{n}\right]} e_{n}
$$

converges for every $f \in \mathcal{H}_{\varphi}$ if and only if there exists a positive constant $\delta$ satisfying: $\left|\left[e_{n}, e_{n}\right]\right| \geq \delta$ for $n \geq 1$.

This can be stated in terms of the theory of Riesz bases. The functions $e_{n}$ form a Riesz basis for $\mathcal{H}_{\varphi}$ if and only if the sequence $\lambda_{n}$ is uniformly separated. From this point of view,

is a biorthogonal sequence to $e_{n}$.

$$
e_{n}^{\prime}:=\frac{C e_{n}}{\left[e_{n}, e_{n}\right]}
$$

If the $\lambda_{n}$ satisfy the Carleson condition, then each $f$ in $\mathcal{H}_{\varphi}$ is given by the interpolation formula

$$
f(z)=\sum_{n=1}^{\infty} \frac{f\left(\lambda_{n}\right)}{\varphi^{\prime}\left(\lambda_{n}\right)} Q_{n}(z)
$$

which converges in norm. This also gives the orthogonal projection from $H^{2}$ onto $\mathcal{H}_{\varphi}$ of any function interpolating the values $f\left(\lambda_{n}\right)$ at the nodes $\lambda_{n}$. 
As in the finite dimensional case, the inner product on $\mathcal{H}_{\varphi}$ has a simple representation in terms of the conjugation operator $C$. In light of the interpolation formula (23) we have

$$
\langle f, g\rangle=\sum_{n=1}^{\infty} \frac{f\left(\lambda_{n}\right) C g\left(\lambda_{n}\right)}{\varphi^{\prime}\left(\lambda_{n}\right)}
$$

for any $f, g$ in $\mathcal{H}_{\varphi}$. The antilinearity in the second argument of the inner product on $\mathcal{H}_{\varphi}$ is clearly reflected by the presence of the $C$ operator in the preceding formula. In some sense, there is an asymmetry in (24) that is unnecessary. We can easily remedy this by considering the bilinear form $[\cdot, \cdot]$, with respect to which $\mathcal{H}_{\varphi}$ is simply a weighted sequence space:

$$
[f, g]=\sum_{n=1}^{\infty} \frac{f\left(\lambda_{n}\right) g\left(\lambda_{n}\right)}{\varphi^{\prime}\left(\lambda_{n}\right)}
$$

Although the bilinear form is not positive definite, we still have

$$
c_{1} \sum_{n=1}^{\infty}\left|\left[f, e_{n}\right]\right|^{2} \leq\|f\|^{2} \leq c_{2} \sum_{n=1}^{\infty}\left|\left[f, e_{n}\right]\right|^{2}
$$

for some constants $c_{1}$ and $c_{2}$ since the $e_{n}$ form a Riesz basis for $\mathcal{H}_{\varphi}$.

\section{INNER MATRICES AND DARLINGTON SYNTHESIS}

In this section we consider a basic matrix extension problem arising in electrical network theory from the viewpoint of $C$-symmetry. We consider the scalar-valued Darlington synthesis problem: Given a function $a(z)$ belonging to $H^{2}$, do there exist functions $b, c$, and $d$ also belonging to $H^{2}$ such that the matrix

$$
U=\left(\begin{array}{cc}
a & -b \\
c & d
\end{array}\right)
$$

is unitary a.e. on the unit circle $\partial \mathbb{D}$ ? In other words, when can we extend the $1 \times 1$ matrix $(a)$ to a $2 \times 2$ inner matrix?

If a matrix $U$ of the form (25) is unitary a.e. on $\partial \mathbb{D}$, then its $\operatorname{determinant~} \operatorname{det} U$ is an inner function, say $\varphi$. It turns out that the entries of $U$ (including $a$ itself) belong to $\mathcal{H}_{z \varphi}$, the backward shift invariant subspace of $H^{2}$ generated by $\varphi$. The following theorem from [21, 22] gives the exact relationship between $a$ and $\operatorname{det} U$.

Theorem 6. If $\varphi$ is a nonconstant inner function, then $U$ is unitary a.e on $\partial \mathbb{D}$ and $\operatorname{det} U=\varphi$ if and only if:

(1) $a, b, c, d$ belong to $\mathcal{H}_{z \varphi}:=H^{2} \ominus z \varphi H^{2}$.

(2) $C a=d$ and $C b=c$.

(3) $|a|^{2}+|b|^{2}=1$ a.e. on $\partial \mathbb{D}$.

Here $C$ denotes the conjugation operator $C f=\bar{f} \varphi$ on the model space $\mathcal{H}_{z \varphi}$ (see Examples 1, 3). From the viewpoint of $C$-symmetry, $2 \times 2$ matrix inner functions resemble quaternions of unit modulus, for

$$
U=\left(\begin{array}{cc}
a & -b \\
C b & C a
\end{array}\right)
$$

where $|a|^{2}+|b|^{2}=1$ a.e. on $\partial \mathbb{D}$. The connection between matrix inner functions and $C$-symmetry is not surprising. Indeed, the connection between the Darlington 
synthesis problem and the backward shift operator (via pseudocontinuations [46]) was noted by several authors [4, 12, 13].

Note that $\|a\|_{\infty} \leq 1$ is necessary for the scalar-valued Darlington synthesis problem with data $a(z)$ to have a solution. The following theorem (also from [21, 22]) is the key to our approach.

Theorem 7. If the function a $(z)$ belongs to $\mathcal{H}_{z \varphi}$ for some nonconstant inner function $\varphi$ and $\|a\|_{\infty} \leq 1$, then there exists a function $b(z)$ in $\mathcal{H}_{z \varphi}$ such that $|a|^{2}+|b|^{2}=1$ a.e. on $\partial \mathbb{D}$.

Returning to Theorem [6, we may write $a=I_{a} F, C a=I_{C a} F, b=I_{b} G$, and $C b=I_{C b} G$ where $I_{a}, I_{C a}, I_{b}, I_{C b}$ are inner functions and $F$ and $G$ are outer. With this notation we have

$$
\begin{aligned}
\varphi & =a C a+b C b \\
& =\mathcal{I}_{F} F^{2}+\mathcal{I}_{G} G^{2},
\end{aligned}
$$

where $\mathcal{I}_{F}$ and $\mathcal{I}_{G}$ denote the associated inner functions (Subsection 7.1) of $F$ and $G$, respectively.

8.1. Primitive solution sets. Suppose that $U$ is a solution to the scalar-valued Darlington synthesis problem with data $a(z)$. By Theorem 6, $\operatorname{det} U=\varphi$ is inner and

$$
U=\left(\begin{array}{cc}
a & -b \\
C b & C a
\end{array}\right)
$$

where $C a$ and $C b$ are the conjugates of $a$ and $b$ in $\mathcal{H}_{z \varphi}$. Observe that if $I_{1}$ and $I_{2}$ are any inner functions, then

$$
U^{\prime}=\left(\begin{array}{cc}
a & -I_{1} b \\
I_{2} C b & I_{1} I_{2} C a
\end{array}\right)
$$

is another solution and $\operatorname{det} U$ divides $\operatorname{det} U^{\prime}$.

We say that a solution $U$ is primitive if the inner function $\varphi=\operatorname{det} U$ is the minimal inner function such that $\operatorname{det} U \operatorname{divides} \operatorname{det} U^{\prime}$ for any other solution $U^{\prime}$. This is equivalent to requiring that $\varphi$ is the minimal inner function such that $a$ belongs to $\mathcal{H}_{z \varphi}$. Note also that every primitive solution shares the same determinant, up to a unimodular constant factor. We call such a $\varphi$ a minimal determinant for the problem (with data $a(z)$ ). Recall that Arov [5, 6] considered a related concept in his classification of minimal $D$-representations in the operator-valued case (which clearly covers the scalar case). Our techniques in the scalar case, however, are completely different, since we have available the concept of determinants and $C$ symmetry. The following easy proposition is from [22].

Proposition 6. Fix a minimal determinant $\varphi$ corresponding to the data a(z). If $U^{\prime}$ is any solution, then $U^{\prime}$ can be obtained via (29) from a primitive solution $U$ with $\operatorname{det} U=\varphi$.

A complete collection of primitive solutions sharing the same minimal determinant is called a primitive solution set. Fix a minimal determinant $\varphi$ to our problem. We wish now to describe all solutions $U$ with determinant $\varphi$. By condition (3) of Theorem [6. we may identify each solution with the inner factor of the upper-left corner $b(z)$. This inner factor must be a divisor of $\mathcal{I}_{G}$ (which is determined by (28)) and hence there is a bijective correspondence between a primitive solution set and the inner divisors of $\mathcal{I}_{G}$. 
Example 14. If $\mathcal{I}_{G}$ is constant, then each primitive solution set consists of precisely one solution and all possible solutions can be constructed via (29) from a single primitive solution. This is the case for the data $a=\frac{1}{2}(1+\varphi)$ where $\varphi$ is an inner function. The minimal determinant is $\varphi$ and the corresponding $b$ is given by $b=\frac{1}{2 i}(1-\varphi)$.

Example 15. If $\mathcal{I}_{G}$ is the square of an inner function, then symmetric primitive solutions exist. By a symmetric solution, we mean here a solution $U$ such that $U=U^{t}$ where $U^{t}$ denotes the transpose of $U$. Observe that if $\mathcal{I}_{G}=I^{2}$ where $I$ is an inner function, then the function $b=I G$ belongs to $\mathcal{H}_{z \varphi}$ and $C b=b$. Using (29) with $I_{1}=-i$ and $I_{2}=i$ gives the symmetric solution

$$
\left(\begin{array}{cc}
a & i b \\
i b & C a
\end{array}\right)
$$

8.2. Rational data. We sketch now an approach (for further details see [22]) to the Darlington problem for rational data based on $C$-symmetry. Given a rational function $a(z)$ (not a finite Blaschke product) in $H^{\infty}$ satisfying $\|a\|_{\infty} \leq 1$, we may write $a=P / R$ where $P(z)$ is a polynomial relatively prime to a polynomial $R(z)$ of the form (19). We consider only the case $\operatorname{deg} P \leq \operatorname{deg} R$ here, the other case is similar.

The data $a(z)$ belongs to $\mathcal{H}_{z \varphi}$ where $\varphi$ denotes the finite Blaschke product

$$
\varphi(z)=\prod_{k=1}^{N} \frac{z-\lambda_{k}}{1-\overline{\lambda_{k}} z} .
$$

The reader will easily verify that the finite Blaschke product $\varphi$ is the minimal determinant corresponding to $a(z)$ and that the $C$ operator on $\mathcal{H}_{z \varphi}$ assumes the form $C(F / R)=F^{\#} / R$ where $F^{\#}=z^{N} \overline{F(1 / \bar{z})}$. In particular, we have $\varphi=R^{\#} / R$ since 1 and $\varphi$ are conjugate functions in $\mathcal{H}_{z \varphi}$.

By Theorem 6 and (27) we seek solutions $U$ of the form

$$
U=\left(\begin{array}{cc}
a & -b \\
C b & C a
\end{array}\right)
$$

where $\varphi=a C a+b C b$. Let us write $b=I_{b} G$ and $C b=I_{C b} G$ where $I_{b}$ and $I_{C b}$ are inner and $G$ denotes the common outer factor of $b$ and $C b$. (27) and (28) imply that

$$
\mathcal{I}_{G} G^{2}=I_{b} I_{C b} G^{2}=b C b=\frac{R^{\#} R-P^{\#} P}{R^{2}}
$$

where $\mathcal{I}_{G}$ denotes the associated inner function for $G$.

Since $G$ belongs to $\mathcal{H}_{z \varphi}$, we have $G=S / R$ where $S(z)$ is a polynomial of degree $\leq n$. Since $G(z)$ and $R(z)$ are outer, the polynomial $S(z)$ is also outer and thus (30) reduces to

$$
\mathcal{I}_{G} S^{2}=R^{\#} R-P^{\#} P
$$

where $\mathcal{I}_{G}$ is a finite Blaschke product (possibly constant). On $\partial \mathbb{D}$ we have

$$
\frac{R^{\#} R-P^{\#} P}{R^{2}}=\varphi\left(1-|a|^{2}\right)
$$

and hence the roots of $R^{\#} R-P^{\#} P$ which lie on $\partial \mathbb{D}$ are exactly the points at which $|a|=1$. Since the zeroes of $R^{\#} R-P^{\#} P$ occur in pairs symmetric with respect to 
$\partial \mathbb{D}$, the number of zeros of $\mathcal{I}_{G}$ (counted according to multiplicity) depends on the degree of $R^{\#} R-P^{\#} P$ and the number of times (according to multiplicity) that the data function $a(z)$ assumes its maximum possible modulus of one on $\partial \mathbb{D}$. The number of solutions in a primitive solution set, therefore, depends on how many times the data $a(z)$ assumes extreme values. Since the Schur-Cohn algorithm [32] can detect the number of zeroes of a polynomial inside the disk, on its boundary, and outside, we can in principle find the number of solutions in a primitive solution set without explicitly finding the roots of polynomials.

We may factor $R^{\#} R-P^{\#} P$ into inner and outer factors without necessarily knowing its zeroes, obtaining $S^{2}$ and hence $S$. This yields the (possibly identical) solutions

$$
\left(\begin{array}{cc}
P / R & -S / R \\
S^{\#} / R & P^{\#} / R
\end{array}\right) \quad \text { and } \quad\left(\begin{array}{cc}
P / R & -S^{\#} / R \\
S / R & P^{\#} / R
\end{array}\right)
$$

to our problem.

Since $G=S / R$ is an outer function in $\mathcal{H}_{z \varphi}$, we have

$$
\widehat{G}=\mathcal{I}_{G} G=\frac{S^{\#}}{R}
$$

and therefore the desired inner function $\mathcal{I}_{G}$ is given by $\mathcal{I}_{G}=S^{\#} / S$. Since $S$ is outer, the zeroes of $\mathcal{I}_{G}$ are precisely the zeros of $S^{\#}$ lying in the open unit disk. Once these zeroes have been found, we can complete our primitive solution set since these solutions can be identified with the functions

$$
b(z)=I_{b} G=I_{b} \frac{S}{R}
$$

where $I_{b}$ is an inner divisor of $\mathcal{I}_{G}$. This yields the following procedure.

Algorithm. Suppose that we are given a rational function (not a finite Blaschke product) $a(z)$ satisfying $\|a\|_{\infty} \leq 1$.

(1) Write $a(z)=P(z) / R(z)$ where $R(z)$ has constant term 1 and $P(z)$ is relatively prime to $R(z)$. Let the degrees of $P$ and $R$ be denoted $m$ and $n$, respectively.

(2) If $m \leq n$, then form the polynomial $R^{\#} R-P^{\#} P$ (of degree at most $2 n$ ) using the definition $F^{\#}(z)=z^{n} \overline{F(1 / \bar{z})}$ for polynomials $F(z)$ of degree $\leq n$.

(a) The outer factor of $R^{\#} R-P^{\#} P$ is a polynomial $S^{2}$ of degree $\leq 2 n$. The matrices

$$
\left(\begin{array}{cc}
P / R & -S / R \\
S^{\#} / R & P^{\#} / R
\end{array}\right) \quad \text { and } \quad\left(\begin{array}{cc}
P / R & -S^{\#} / R \\
S / R & P^{\#} / R
\end{array}\right)
$$

are primitive solutions with determinant $\varphi=R^{\#} / R$.

(b) Find the roots of the polynomial

$$
S^{\prime}:=\frac{S^{\#}}{\operatorname{gcd}\left(S, S^{\#}\right)}
$$

(of degree $N \leq n$ ). These zeroes all lie inside the unit disk. 
(c) For each subset $\left\{\omega_{1}, \ldots, \omega_{k}\right\}$ of the roots of $S^{\prime}$ such that $k \leq\left\lfloor\frac{N}{2}\right\rfloor$,

$$
T(z)=S(z) \prod_{j=1}^{k} \frac{z-\omega_{j}}{1-\overline{\omega_{j}} z}
$$

is a polynomial of degree $N-k$ yielding the primitive solutions

$$
\left(\begin{array}{cc}
P / R & -T / R \\
T^{\#} / R & P^{\#} / R
\end{array}\right) \quad \text { and } \quad\left(\begin{array}{cc}
P / R & -T^{\#} / R \\
T / R & P^{\#} / R
\end{array}\right) .
$$

This yields a complete set of primitive solutions with determinant $\varphi$.

(3) If $m>n$, then form the polynomial $R^{\#} R-P^{\#} P$ (of degree at most $2 m$ ) using the definition $F^{\#}(z)=z^{m} \overline{F(1 / \bar{z})}$ for polynomials $F(z)$ of degree $\leq m$. Proceed as in the previous case.

\section{INVARIANT SUBSPACES OF $C$-SYMMETRIC OPERATORS}

We now briefly consider invariant subspaces of $C$-symmetric operators. In particular, we are primarily interested in subspaces that are simultaneously invariant under a $C$-symmetric operator $T$ and the underlying involution $C$.

The proof of the following proposition is left to the reader.

Proposition 7. Let $(\mathcal{H}, T, C)$ denote a $C$-symmetric triple.

(1) $\mathcal{M}$ is $C$-invariant if and only if $\mathcal{M}^{\perp}$ is $C$-invariant.

(2) If $\mathcal{M}$ is a subspace of $\mathcal{H}$ that is invariant under $C$ and $T$, then $\mathcal{M}$ reduces $T$.

(3) $\mathcal{M}$ reduces $T$ if and only if $C \mathcal{M}$ reduces $T$.

(4) If $\mathcal{M}$ is a $C$ invariant subspace of $\mathcal{H}$ and $P$ denotes the orthogonal projection from $\mathcal{H}$ onto $\mathcal{M}$, then the compression $A=P T P$ of $T$ to $\mathcal{M}$ satisfies $C A=A^{*} C$.

Example 16. Consider the $C$-symmetric triple $\left(\mathbb{C}^{n}, J_{n}(\lambda), C\right)$ of Example 4 There are no proper, nontrivial subspaces of $\mathbb{C}^{n}$ that are simultaneously invariant for both the Jordan block $J:=J_{n}(\lambda)$ and the involution $C$. If $\mathcal{M}$ is a nontrivial subspace of $\mathbb{C}^{n}$ which is $J$-invariant, then it must contain the vector $(1,0, \ldots, 0)$. However, $C(1,0, \ldots, 0)=(0, \ldots, 0,1)$ and inductively one can see that if $\mathcal{M}$ is also $C$-invariant, then $\mathcal{M}$ must be all of $\mathbb{C}^{n}$.

Example 17. Consider the $C$-symmetric triple $\left(L^{2}[0,1], V, C\right)$ of Subsection 4.3 , It is well known that the only invariant subspaces for the Volterra integration operator are the subspaces $\chi_{[0, a)} L^{2}[0,1]$ where $a \in[0,1]$ and $\chi_{[0, a)}$ denotes the characteristic function of the interval $[0, a)$. It is clear that there are no proper, nontrivial $V$ invariant subspaces of $L^{2}[0,1]$ that are also $C$-invariant.

Example 18. We return to the notation of Example 1. There are no proper nontrivial subspaces of $\mathcal{H}_{\varphi}$ which are invariant under both $C$ and the backward shift operator $B$. Restricted to $\mathcal{H}_{\varphi}, B$ is simply the compression to $\mathcal{H}_{\varphi}$ of the Toeplitz operator $T_{\bar{z}}$ and hence, by Proposition [3, $B$ is $C$-symmetric. Suppose that $\mathcal{M}$ is a subspace of $\mathcal{H}_{\varphi}$ that is invariant for both $B$ and $C$. Without loss of generality, there exists a function $f$ in $\mathcal{M}$ with a nonconstant outer factor, say $F$. The function $g:=F+C F$ belongs to $\mathcal{M}$ and satisfies $C g=g$. Since $F$ and $C F$ 
share the same outer factor, namely $F$, the function $g$ itself is outer. However, a selfconjugate outer function in $\mathcal{H}_{\varphi}$ must generate $\mathcal{H}_{\varphi}$ by a proposition in [21] (which is a simple restatement of [14, Theorem 3.1.5] in terms of conjugation operators).

Despite these examples, there are many $C$-symmetric triples $(\mathcal{H}, T, C)$ such that $\mathcal{H}$ has subspaces that are invariant for both $T$ and $C$. For instance, if the matrix representation for $T$ with respect to the basis $e_{n}$ furnished by Lemma 11 has a diagonal block, then $T$ clearly has a subspace that is simultaneously invariant for $T$ and $C$. Finally, we note in passing that Theorem 2 immediately implies that the antilinear operators $C T$ and $T C$ always admit nontrivial invariant subspaces.

\section{Conjugation in SEVERAl COMPlex VARIABles}

This section is concerned with the analogue of Jordan operators in several complex variables and, in particular, the question whether they can still be $C$ symmetric. The following observation can produce many examples of product $C$ symmetric operators.

Lemma 6. Let $\left(\mathcal{H}_{1}, T_{1}, C_{1}\right)$ and $\left(\mathcal{H}_{2}, T_{2}, C_{2}\right)$ be $C$-symmetric triples. Then the hilbertian tensor product $\left(\mathcal{H}_{1} \otimes \mathcal{H}_{2}, T_{1} \otimes T_{2}, C_{1} \otimes C_{2}\right)$ is also a $C$-symmetric triple.

However, the general picture on an analytic model space on the polydisk is more involved. To start with such a product example, let

$$
\Phi\left(z_{1}, z_{2}, \ldots, z_{n}\right)=\varphi_{1}\left(z_{1}\right) \varphi_{2}\left(z_{2}\right) \ldots \varphi_{n}\left(z_{n}\right)
$$

be a product of inner functions in the respective variables. The function $\Phi$ is inner in the polydisk and a standard algebraic argument shows that

$$
\mathcal{K}_{1}=H^{2}\left(\mathbf{D}^{n}\right) \ominus \sum_{k=1}^{n} \varphi_{k} H^{2}\left(\mathbf{D}^{n}\right) \cong \bigotimes_{k=1}^{n}\left(H^{2} \ominus \varphi_{k} H^{2}\right) .
$$

The associated product conjugation on this space is

$$
C f(z)=\varphi_{1}\left(z_{1}\right) \ldots \varphi_{n}\left(z_{n}\right) \overline{\left[z_{1} \ldots z_{n} f(z)\right]},
$$

as a direct computation can also verify that $C f$ is jointly analytic and orthogonal to $\sum_{k=1}^{n} \varphi_{k} H^{2}\left(\mathbf{D}^{n}\right)$. Let $f \in C\left(\overline{\mathbf{D}^{n}}\right)$ be a continuous function and let $\mathrm{T}_{f}$ be the Toeplitz operator with symbol $f$ compressed to the space $\mathcal{K}_{1}$. Since the function $f$ is approximable in the uniform norm by real analytic monomials, the above lemma implies $C \mathrm{~T}_{f}=\mathrm{T}_{f}^{*} C$.

On the other hand, let us consider the model space $\mathcal{K}_{2}=H^{2}\left(\mathbf{D}^{2}\right) \ominus z_{1} z_{2} H^{2}\left(\mathbf{D}^{2}\right)$. The orthogonal decomposition

$$
H^{2}\left(\mathbf{D}^{2}\right) \ominus z_{1} z_{2} H^{2}\left(\mathbf{D}^{2}\right) \cong H^{2}\left(\mathbf{D}_{1}\right) \oplus z_{2} H^{2}\left(\mathbf{D}_{\mathbf{2}}\right),
$$

holds, where the subscripts indicate the corresponding variable. Thus the compressed Toeplitz operator $T_{z_{1}}$ is unitarily equivalent to the standard unilateral shift $T_{z}$ and therefore has nontrivial Fredholm index. Consequently, the model space $\mathcal{K}_{2}$ cannot carry an involution $C$ with respect to which the respective Jordan operators are $C$-symmetric.

One may ask what properties distinguish the quotient analytic modules $\mathcal{K}_{1}$ and $\mathcal{K}_{2}$ so that the compressed Toeplitz operators are $C$-symmetric on one, but not on the other. 


\section{ACKNOWLEDGMENTS}

We are much indebted to D.Z. Arov, J.W. Helton, S. Richter, D. Sarason, H.S. Shapiro, and C. Sundberg for their interest and constructive comments on different parts of this work, at different stages of its evolution. We also thank the anonymous referee for valuable bibliographical references.

\section{REFERENCES}

[1] Akhiezer, N.I., The Classical Moment Problem, Oliver and Boyd, Edinburgh, 1965. MR0184042(32:1518)

[2] Alpay, D., Dijksma, A., Rovnyak, J., de Snoo, H., Schur functions, operator colligations, and reproducing kernel Pontryagin spaces, Birkhäuser, Basel, 1997. MR.1465432 (2000a:47024)

[3] Ahern, P., Clark, D., On inner functions with $H^{p}$ derivative, Michigan Math. J. 21 (1974), 115-127. MR0344479(49"9218)

[4] Arov, D.Z., Darlington's method in the study of dissipative systems, Dokl. Akad. Nauk SSSR 201 (1971), no. 3, 559-562. MR0428098 (55:1127)

[5] Arov, D.Z., Stable dissipative linear stationary dynamical scattering systems, Operator Theory: Advances and Applications, 134 (2002), 99-136. MR.2013544 (2004j:47016)

[6] Arov, D.Z., Realization of matrix-valued functions according to Darlington, Math USSR Izvestija, 7(6) (1973), 1295-1326. MR0357820 (50:10287)

[7] Asadi, S., Lucenko, I. E., Antiunitary transformations of linear operators (Russian), Vestnik Harkov. Gos. Univ. No. 83 Mat. i Meh. Vyp. 37 (1972), 13-20, 120. MR0333778 (48:12102)

[8] Bercovici, H., Operator Theory and Arithmetic in $H^{\infty}$, Amer. Math. Soc., Providence, R.I., 1988. MR0954383 (90e:47001)

[9] Bergman, S., Schiffer, M., Kernel functions and conformal mapping, Composition Math. 8 (1951), 205-249. MR0039812 (12:602c)

[10] Cima, J.A., Ross, W.T., The Backward Shift on the Hardy Space, American Mathematical Society, Providence R.I., 2000. MR.1761913 (2002f:47068)

[11] Clark, D.N., One dimensional perturbations of restricted shifts, J. Anal. Math. 25, (1972) 169-191. MRR0301534 (46:692)

[12] deWilde, P., Roomy scattering matrix synthesis, Technical Report (Berkeley, 1971).

[13] Douglas, R.G., Helton, J. William, Inner dilations of analytic matrix functions and Darlington synthesis, Acta Sci. Math (Szeged), 34 (1973), 61-67. MR0322538(48:900)

[14] Douglas, R.G., Shapiro, H.S., Shields, A.L., Cyclic vectors and invariant subspaces for the backward shift operator, Ann. Inst. Fourier (Grenoble) 20 no. 1 (1970), 37-76. MR 0270196 $(42: 5088)$

[15] Duren, P.L., Theory of $H^{p}$ Spaces, Pure and Appl. Math., Vol 38, Academic Press, New York, 1970. MR0268655 (42:3552)

[16] Foiaş, C., Frazho, A.E., The commutant lifting approach to interpolation problems, Birkhäuser Verlag, Basel, 1990. MR1120546 (92k:47033)

[17] Friedrichs, K., On certain inequalities for analytic functions and for functions of two variables, Trans. Amer. Math. Soc. 41 (1937), 321-364. MR.1501907

[18] Gantmacher, F.R., The theory of matrices, Volume II, Chelsea, New York, 1989. MR0107649 $(21: 6372 \mathrm{c})$

[19] Gantmacher, F.R., Krein, M.G., Oscillation Matrices and Kernels and Small Vibrations of Mechanical Systems (Revised English Edition), American Math. Soc. Chelsea Publ., Amer. Math. Soc., Providence, R.I., 2002. MR.1908601 (2003f:34161)

[20] Garcia, S.R., A*-closed subalgebra of the Smirnov class, Proc. Amer. Math. Soc. 133 (2005), 2051-2059.

[21] Garcia, S.R., Conjugation, the backward shift, and Toeplitz kernels, to appear: Journal of Operator Theory.

[22] Garcia, S.R., Inner matrices and Darlington synthesis, to appear: Methods Funct. Anal. Topology.

[23] Garcia, S.R., Sarason, D., Real outer functions, Indiana Univ. Math. J., 52 (2003), 1397-1412. MR2021044 (2004k:30129)

[24] Glazman, I.M., Direct methods of the qualitative spectral theory of singular differential operators (Russian), Gos. Iz. Fiz.-Mat. Lit., Moscow, 1963. MR0185471 (32:2938) 
[25] Godic, V.I., Lucenko, I.E., The structure of bisymmetric operators (Russian), Teoria Funkt. Funktional. Anal. Prilozh. 16 (1972), 138-139. MR0344934 (49:9673)

[26] Gohberg, I., Krein, M.G., Introduction to the Theory of Linear Nonselfadjoint Operators in Hilbert Space, Amer. Math. Soc., Providence, R.I., 1969. MR0246142 (39:7447)

[27] Gohberg, I., Krein, M.G., Theory and applications of Volterra operators in Hilbert space, Amer. Math. Soc., Providence, R.I., 1971. MR0264447 (41:9041)

[28] Gorbachuk, M.L., Gorbachuck, V.I., M.G.Krein's Lectures on Entire Operators Birkhäuser, Basel, 1997. MR.1466698 (99f:47001)

[29] Hamburger, H.L., Uber die Zerlegung des Hilbertschen Raumes durch vollstetige lineare Transformationen, Math. Nachr. 4 (1951), 56-69. MR.0040587 (12:718b)

[30] Horn, R.A., Johnson, C.R., Matrix Analysis, Cambridge Univ. Press, Cambridge, 1985. MR0832183 (87e:15001)

[31] Kato, T., Perturbation theory for linear operators, Springer-Verlag, Berlin, 1995. MR1335452 (96a:47025)

[32] Krein, M.G., Naimark, M.A., The method of symmetric and Hermitian forms in the theory of the separation of the roots of algebraic equations, Linear and Multilinear Algebra 10 (1981), no. 4, 265-308. MR0638124 (84i:12016)

[33] Helgason, S., Differential geometry, Lie groups, and symmetric spaces, Academic Press, Boston, 1978. MR0514561 (80k:53081)

[34] Helson, H., Large analytic functions, II., Analysis and Partial Differential Equations, Lecture Notes in Pure and Appl. Math., 122, Dekker, New York, 1990, 217-220. MR1044789 (92c:30039)

[35] Lotto, B.A., Sarason, D., Multiplicative structure of de Branges's spaces, Rev. Mat. Iberoamericana 7 (1991), 183-220. MR:1133377 (92k:46035)

[36] Lucenko, I. E., Linear operators that commute with antiunitary operators (Russian), Teor. Funkt. Funktional. Anal. Prilozen. Vyp., 9 (1969), 85-93. MR0278085 (43:3817)

[37] von Neumann, J., Allgemeine Eigenwerttheorie Hermitischer Funktionaloperatoren, Math. Ann. 102 (1929), 49-131.

[38] Nikolski, N.K., Treatise on the Shift Operator, Springer-Verlag, New York, 1986. MR0827223 (87i:47042)

[39] Nikolski, N.K., Operators, Functions, and Systems: An Easy Reading, Volume 2: Model Operators and Systems, American Mathematical Society, Providence R.I., 2002. MR 1892647 (2003i:47001b)

[40] Peller, V.V., Hankel Operators and Their Applications, Springer Monographs in Mathematics, Springer-Verlag, 2003. MR.1949210 (2004e:47040)

[41] Potapov, V.P., The multiplicative structure of J-contractive matrix functions (in Russian), Trudy Moskov. Mat. Obsc. 4 (1955), 125-236. MR0076882 (17:958f)

[42] Putinar, M., Shapiro, H.S., The Friedrichs operator of a planar domain, I., "S. A. Vinogradov Memorial Volume" (V. Havin, N. K. Nikolskii, eds.), Birkhäuser Verlag, Basel et al., 2000, 303-330; Part II., "Béla Sz.-Nagy Memorial Volume" (L. Kérchy et al., eds.), Birkhäuser, Basel, 2001, 519-551. MR.1771771 (2001g:47049) MR1902820(2003e:47054)

[43] Reed, M., Simon, B., Methods of Modern Mathematical Physics II: Fourier Analysis, SelfAdjointness, Academic Press, New York, 1975. MR0493419 (58:12429a)

[44] Richter, S., Sundberg, C., A formula for the local Dirichlet integral, Michigan Math. J. 38 (1991), 355-379. MR1116495 (92i:47035)

[45] Riesz, F., Sz.-Nagy, B., Functional Analysis, Dover, New York, 1990. MR.1068530(91g:00002)

[46] Ross, W.T., Shapiro, H.S., Generalized Analytic Continuation, University Lecture Series, Volume 25, American Mathematical Society, Providence R.I., 2002. MR.1895624 (2003h:30003)

[47] Rudin, W., Function theory in the polydisk, W.A. Benjamin, New York, 1969. MR0255841 $(41: 501)$

[48] M. Schiffer, Fredholm eigenvalues and Grunsky matrices, Ann. Polonici Math. 39 (1981), 149- 164. MR0617457 (82k:30010)

[49] Siegel, C.L., Symplectic geometry, Amer. J. Math. 67 (1943), 1-86. MR0008094 (4:242b)

[50] Simon, B., Spectral analysis of rank one perturbations and applications, in vol. Mathematical quantum theory. II. Schrödinger operators (Vancouver, BC, 1993), pp. 109-149, CRM Proc. Lecture Notes 8, Amer. Math. Soc., Providence, RI, 1995. MR.1332038 (97c:47008)

[51] Takagi, T., On an algebraic problem related to an analytic theorem of Caratheodory and Fejer and on an allied theorem of Landau, Japan J. Math. 1 (1925), 83-93. 
[52] Taussky, O., The role of symmetric matrices in the study of general matrices, Linear Algebra Appl. 5 (1972), 147-154. MR0302674 (46:1818)

[53] Thompson, J., Approximation in the mean by polynomials, Ann. Math. 133 (1991), 477-507. MR:1109351 (93g:47026)

Department of Mathematics, University of California at Santa Barbara, Santa BarBara, CALifornia 93106-3080

E-mail address: garcias@math.ucsb.edu

$U R L:$ http://math.ucsb.edu/ garcias

Department of Mathematics, University of California at Santa Barbara, Santa BarBara, CALIFornia 93106-3080

E-mail address: mputinar@math.ucsb.edu

URL: http://math.ucsb.edu/ ${ }^{\text {mputinar }}$ 